Homology, Homotopy and Applications, vol.9(1), 2007, pp.1-43

\title{
HIGHER ORDER COHOMOLOGY OPERATIONS AND MINIMAL ATOMICITY
}

\author{
ROCHELLE PEREIRA
}

(communicated by Jesper Grodal)

\begin{abstract}
We prove that $\Omega S_{(2)}^{n}, S^{n}\left\{2^{r}\right\}$, and $\Omega^{2} S_{(2)}^{n}$ are minimal atomic spaces for appropriate values of $n$. We do this by using secondary and tertiary cohomology operations to prove that, above the Hurewicz dimension, no elements in the mod 2 homology of the cited spaces are in the image of the Hurewicz homomorphism. In the case of $\Omega^{2} S^{n}$, we construct and exploit an appropriate filtration to facilitate the use of higher order cohomology operations. An appendix consisting of an examination of the coefficients in Adams' factorization is included.
\end{abstract}

\section{Introduction}

In this document, we study minimal atomic spaces, defined here in Section 2, at the prime 2 . Introduced in $[\mathbf{H K M}]$ by $\mathrm{Hu}$, Kriz, and May, minimal atomicity is a natural derivative of the atomicity concept which has been pervasive in the literature $[\mathbf{A K}],[\mathbf{B M}],[\mathbf{C M N}],[\mathbf{H K M}],[\mathbf{X}]$. Baker and May studied minimal atomicity more extensively in $[\mathbf{B M}]$ with an appendix by the author. The authors restricted themselves to Hurewicz complexes, $p$-local CW spaces whose first non-trivial homotopy group is a cyclic module over $\mathbb{Z}_{(p)}$. The main result we use from that paper is its characterization of minimal atomic spaces as those Hurewicz complexes which have no homotopy detected by mod 2 homology. This criterion is verified by showing that the primitive elements of mod 2 homology fail to be in the image of the Hurewicz homomorphism.

Baker and May show that minimal atomic spaces are common; they provide a method for constructing a minimal atomic space from any atomic space. Yet, explicit examples of minimal atomic spaces are few. (Baker and May do provide explicit examples of minimal atomic spectra.) We show that the techniques of higher order cohomology operations can be applied to prove that a space is minimal atomic. This technique has unearthed a new minimal atomic space, $S^{n}\left\{2^{r}\right\}$, and reestablished minimal atomicity of $\Omega S_{(2)}^{n}$ and $\Omega^{2} S_{(2)}^{n}$ for certain values of $n$ and $r$.

Main Theorem. Let $n$ be a positive integer greater than 1. Higher order cohomology operations can be defined on the following spaces and used to show that they are minimal atomic:

Received January 16, 2006, revised August 25, 2006; published on November 3, 2006.

2000 Mathematics Subject Classification: 55S20.

Key words and phrases: minimal atomic, cohomology operations.

Copyright (c) 2006, International Press. Permission to copy for private use granted. 
(i) $\Omega S_{(2)}^{n}$, for $n \neq 2,4,8$.

(ii) $S^{n}\left\{2^{r}\right\}$, for $r>1$ and $n \neq 2^{s}$ for any $s$.

(iii) $\Omega^{2} S_{(2)}^{n}$, for $n \neq 2,3,4,5,8,9$.

After isolating those primitive elements which could be spherical, we show that the dual indecomposable elements are in the target of a higher order operation. Thus, none of these primitive elements are spherical. In most cases, secondary cohomology operations will suffice, but occasionally we must appeal to tertiary cohomology operations to do the job.

The organization of this document is as follows. In Section 2, after reviewing some definitions, we give a proof using Hopf Invariant One of the minimal atomicity of $\Omega S^{n}$ for $n \neq 1,2,4,8$ and outline the essence of the higher cohomology operation argument. Section 3 lays out the background of the higher order cohomology operations we use: the Brown-Peterson secondary cohomology operations, Adams' Hopf Invariant One secondary cohomology operations, and a tertiary cohomology operation which is defined using Adams' factorization of $S q^{2^{r+1}}$. These operations are used to establish $\Omega S_{(2)}^{n}$ is minimal atomic when $n \neq 1,2,4,8$ in Section 4 . These higher order cohomology operation proofs pave the way for an analogous proof that $S^{n}\left\{2^{r}\right\}$ is minimal atomic in Section 5 as well as an examination of why these methods seem unable to show that $S^{n}\left\{2^{r}\right\}$ is minimal atomic when $n$ is a power of 2 . In Section 6 a filtration of $\Omega^{2} S_{(2)}^{n}$ is developed based on the James construction filtration of $\Omega S_{(2)}^{n}$. This filtration, along with secondary and tertiary cohomology operation arguments, shows that $\Omega^{2} S_{(2)}^{n}$ is minimal atomic in Section 7 . The document ends with an appendix which discusses one computer program to obtain the aforementioned factorization of $S q^{2^{r+1}}$.

Beginning with Section 2.2, spaces will be localized at the prime 2 unless otherwise specified, and all homology and cohomology will be taken with $\mathbb{F}_{2}$ coefficients; $n$ will always denote a positive integer with $n \neq 1,2,4,8$ unless otherwise specified. The notation $K(\pi, m)$ denotes an Eilenberg-MacLane space, and $K(m)$ denotes the Eilenberg-MacLane space $K(\mathbb{Z} / 2 \mathbb{Z}, m)$.

\section{Strategies for proving minimal atomicity}

In this section, we review the definitions related to the study of minimal atomicity. The section which follows covers the main ideas used in employing a higher order cohomology operations proof of minimal atomicity. The underpinning for all of these arguments is found in Theorem 2.6, which allows us to assess if a space is minimal atomic given information about which elements of its homology are spherical.

\subsection{Definitions}

We recall those definitions which were specified in $[\mathbf{B M}]$ that are relevant to this document. For a fixed prime $p$, minimal atomic spaces, $X$, must be $p$-local $\mathrm{CW}$ spaces in which the attaching maps are based maps whose domains are spheres localized at the prime $p$. All spaces $X$ we consider must be simply-connected and localized at this prime $p$. The definitions below assume $X$ satisfies these conditions. 
Definition 2.1. Suppose $X$ has the property that $X$ is $\left(n_{0}-1\right)$-connected, but $X$ is not $n_{0}$-connected. The Hurewicz dimension of $X$ is $n_{0}$. If $\pi_{n_{0}}(X)$ is a cyclic module over $\mathbb{Z}_{(p)}, X$ is a Hurewicz complex.

Definition 2.2. Suppose $X$ and $Y$ are Hurewicz complexes with Hurewicz dimension $n_{0}$. Let $f: Y \rightarrow X$ be such that $f_{*}: \pi_{n_{0}}(Y) \otimes \mathbb{F}_{p} \rightarrow \pi_{n_{0}}(X) \otimes \mathbb{F}_{p}$ is an isomorphism and all $f_{*}: \pi_{n}(Y) \rightarrow \pi_{n}(X)$ are monomorphisms. Then $f$ is a monomorphism of Hurewicz complexes.

Definition 2.3. Suppose $X$ is a Hurewicz complex with Hurewicz dimension $n_{0}$. Further, assume any self-map $f: X \rightarrow X$ which induces an isomorphism on $\pi_{n_{0}}(X)$ is an equivalence. Then $X$ is atomic.

Definition 2.4. Suppose $X$ is atomic. Then $X$ is minimal atomic if any monomorphism $f: Y \rightarrow X$, with $Y$ an atomic complex, is an equivalence.

Definition 2.5. Suppose $X$ is a Hurewicz complex and the mod $p$ Hurewicz homorphism $h: \pi_{n}(X) \rightarrow H_{n}\left(X ; \mathbb{F}_{p}\right)$ is zero for all $n>n_{0}$. Then $X$ has no homotopy detected by mod p homology.

The main result we use from $[\mathbf{B M}]$ is:

Theorem 2.6. $X$ is a minimal atomic space if and only if it is has no homotopy detected by mod p homology.

\subsection{James maps}

Before we begin to use higher order cohomology operations, for completeness we recollect a proof that $\Omega S^{n}$ is minimal atomic which does not use higher order cohomology operations. The author is grateful to Fred Cohen for making her aware of this proof.

We have already alluded to the James construction on $X$ denoted $J(X)$ which is equivalent to $\Omega \Sigma X$. We label the $k$ th filtration of the James construction by $J_{k}(X)=X^{k} / \sim$ where

$$
\left(x_{1}, \ldots, x_{j-1}, *, x_{j+1}, \ldots, x_{k}\right) \sim\left(x_{1}, \ldots, x_{j-1}, x_{j+1}, \ldots, x_{k}\right) .
$$

We utilize the James maps,

$$
h_{q}: J(X) \rightarrow J\left(X^{(q)}\right)
$$

where

$$
X^{(q)}=\underbrace{X \wedge \cdots \wedge X}_{q} .
$$

Fred Cohen has pointed out that manipulating the James-Hopf map $h_{2}$ gives us one way to finish proving $\Omega S^{n}$ is minimal atomic. Let us denote the primitive elements of $H_{*}\left(\Omega S^{n}\right)$ by $\left(x_{n-1}\right)^{2^{q}}$. Now, $h_{2}: \Omega S^{n} \rightarrow \Omega S^{2 n-1}$ maps $\left(x_{n-1}\right)^{2^{q}}$ to $\left(x_{2 n-2}\right)^{2^{q-1}}$ in homology. If $\left(x_{n-1}\right)^{2^{q}}$ is spherical, then so is $\left(x_{2 n-2}\right)^{2^{q-1}}$. It follows that $x_{2^{q-1}(n-1)}^{2}$ 
is spherical. We shall show $\left(x_{m}\right)^{2}$ is not spherical for $m \neq 1,3,7$. Then, Theorem 2.6 allows us to conclude that $\Omega S^{n}$ is minimal atomic for $n \neq 2,4,8$.

For any $m$, let $S^{m} \stackrel{\eta}{\rightarrow} \Omega S^{m+1}$ be the unit of the $(\Sigma, \Omega)$ adjunction. Then $i_{m} \in$ $H_{m}\left(S^{m}\right)$ maps to $x_{m}$ under $\eta_{*}$. Suppose $\left(x_{m}\right)^{2}$ is spherical. Then, there exists a nontrivial map $S^{2 m} \rightarrow \Omega S^{m+1}$ such that $\left(x_{m}\right)^{2}$ lies in the image of the map induced by homology. We may take the adjoint of this map to yield $S^{2 m+1} \rightarrow S^{m+1}$, and we may loop this map to obtain $\Omega S^{2 m+1} \rightarrow \Omega S^{m+1}$. By taking the cartesian product of this map with $\eta$ we obtain

$$
S^{m} \times \Omega S^{2 m+1} \rightarrow \Omega S^{m+1} \times \Omega S^{m+1} \stackrel{\mu}{\rightarrow} \Omega S^{m+1}
$$

where $\mu$ is the multiplication map. Then under the composition of maps in (2.1) we have,

$$
i_{m} \otimes\left(x_{2 m}\right)^{i} \rightarrow x_{m} \times\left(x_{m}\right)^{2 i} \rightarrow\left(x_{m}\right)^{2 i+1}
$$

and

$$
1 \otimes\left(x_{2 m}\right)^{i} \rightarrow 1 \times\left(x_{2 m}\right)^{2 i} \rightarrow\left(x_{m}\right)^{2 i} .
$$

We thus have an isomorphism in homology, and the composite of maps in (2.1) is an equivalence. In particular, $S^{m}$ is a retract of $\Omega S^{m+1}$. However, a retract $X$ of an $H$-space $Y$ is an $H$-space via the following commutative diagram:

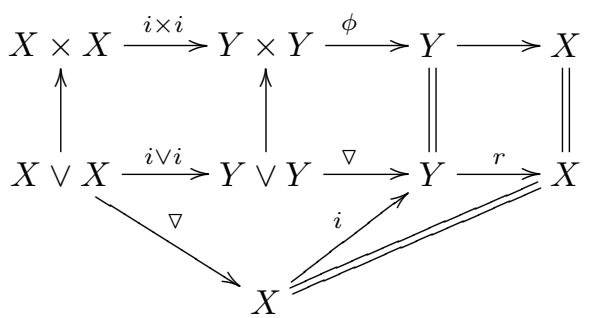

Thus $S^{m}$ must be an $H$-space and so $m=1,3,7$. Recall that if $\left(x_{n-1}\right)^{2^{q}}$ is spherical, $\left(x_{2^{q-1}(n-1)}\right)^{2}$ is spherical. Thus, from above, $2^{q-1}(n-1)=1,3,7$. If $q \geqslant 2$ we obtain a contradiction, so it must be the case that $q=1$ and $n-1=1,3,7$. Thus the only possible spherical elements which exist above the Hurewicz dimension for $\Omega S^{n}$ are the classes $\left(x_{1}\right)^{2} \in H_{2}\left(\Omega S^{2}\right),\left(x_{3}\right)^{2} \in H_{6}\left(\Omega S^{4}\right)$, and $\left(x_{7}\right)^{2} \in H_{14}\left(\Omega S^{8}\right)$. Looping the Hopf maps $\eta: S^{3} \rightarrow S^{2}, \nu: S^{7} \rightarrow S^{4}$, and $\sigma: S^{15} \rightarrow S^{7}$ shows that these elements are spherical. Thus, $\Omega S^{n}$ is minimal atomic if and only if $n \neq 2,4,8$.

\subsection{The higher order cohomology argument}

Theorem 2.6 verifies that a space $X$ is minimal atomic if no spherical elements of $H_{*}(X)$ exist above the Hurewicz dimension. Thus, the first step in the higher order cohomology argument is to calculate which primitive elements of $H_{*}(X)$ with dimension above the Hurewicz dimension are annihilated by the Steenrod algebra; all spherical elements we are interested in must satisfy these properties. We show that each of these candidates cannot be in the image of the Hurewicz homomorphism by using higher order cohomology operations and a naturality argument as follows. 
Let $a \in H_{i}(X)$ be a spherical candidate with dual indecomposable element $\alpha \in H^{i}(X)$. To show $a$ is not spherical, we will prove there exists $\beta \in H^{j}(X)$, with $j \neq i$, and a higher order cohomology operation $\Phi: H^{j}(X) \rightarrow H^{i}(X) / Q_{\Phi}^{i}(X)$ such that

$$
\Phi(\beta)=\alpha+\gamma \neq 0
$$

Here $\gamma \in H^{i}(X)$ is a decomposable element which is possibly zero, and $Q_{\Phi(X)}^{i}$ is a submodule of $H^{i}(X)$ which is possibly zero, in which case $\Phi$ is defined "with zero indeterminacy". Some arguments will attest to the fact that $\Phi$ is defined on $\beta$ and $X$.

For dimensional reasons, $\Phi$ will be defined on all spheres and will evaluate to zero with "zero indeterminacy"; in particular, $\Phi$ will be defined on $S^{i}$. Now, suppose that $a$ is spherical. Then, there must exist a non-trivial map

$$
f: S^{i} \rightarrow X
$$

such that $f^{*}$ maps $\alpha$ to the non-zero element of $H^{i}\left(S^{i}\right)$. Since our higher order cohomology operations are natural with respect to maps, we have the following commutative diagram:

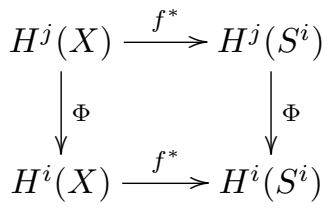

We see that $\left(f^{*} \circ \Phi\right)(\beta)=\alpha$ (modulo zero) while $\left(\Phi \circ f^{*}\right)(\beta)=0$ (modulo zero). We have a contradiction, and we may conclude that $a$ is not spherical. This basic argument will appear throughout the document.

\section{Three kinds of higher order cohomology operations}

We examine the secondary and tertiary cohomology operations which we will utilize. We recall the construction of the Brown-Peterson secondary cohomology operation which is based on a relation in the Steenrod algebra and prove such an operation has stable properties. We review Adams' secondary cohomology operations $\Phi_{i j}$, pointing out similarities and differences with the Brown-Peterson operations. Finally, we construct a tertiary cohomology operation in the manner suggested by [BP] using Adams' factorization of $S q^{2^{r+1}}$ into secondary cohomology operations.

\subsection{Brown-Peterson secondary cohomology operations}

Each secondary cohomology operation stems from a relation in the Steenrod algebra. We recall a particular secondary cohomology operation defined by Brown and Peterson in $[\mathbf{B P}]$. 
Homology, Homotopy and Applications, vol. 9(1), 2007

Suppose for some fixed $m$ we have a factorization of Steenrod operations

$$
S q^{m}=\sum_{i} S q^{a_{i}} S q^{b_{i}}
$$

where each $S q^{a_{i}}, S q^{b_{i}}$ has degree greater than 0 . Let $\kappa_{m}$ be the fundamental class of $K(m)$. For each Steenrod operation $S q^{b_{i}}$, let $S q^{b_{i}}: K(m) \rightarrow K\left(m+b_{i}\right)$ represent the element $S q^{b_{i}}\left(\kappa_{m}\right)$. Then, define $f_{1}: K(m) \rightarrow \prod_{i} K\left(m+b_{i}\right)$ such that $f_{1}^{*}$ takes the fundamental classes of $\prod_{i} K\left(m+b_{i}\right)$ to the elements $S q^{b_{i}}\left(\kappa_{m}\right)$. Denote the homotopy fiber of $f_{1}$ by $A_{1}$, and let the fibration $A_{1} \rightarrow K(m)$ be $g_{1}$. Let

$$
h: \prod_{i} K\left(m+b_{i}-1\right) \rightarrow A_{1}
$$

be the map of the fiber of $g_{1}$ into $A_{1}$. Note that the fundamental classes $\kappa_{m+b_{i}-1}$ transgress to $S q^{b_{i}}\left(\kappa_{m}\right)$ in the Serre spectral sequence of the fibration given by $h \circ g_{1}$. Then, $\sum S q^{a_{i}}\left(\kappa_{m+b_{i}-1}\right)$ transgresses to

$$
\sum S q^{a_{i}} S q^{b_{i}}\left(\kappa_{m}\right)=S q^{m}\left(\kappa_{m}\right)=\kappa_{m}^{2} \neq 0 .
$$

If we loop our fibration, we obtain the new fibration

$$
\prod_{i} K\left(m+b_{i}-2\right) \stackrel{\Omega h}{\longrightarrow} \Omega A_{1} \stackrel{\Omega g_{1}}{\longrightarrow} K(m-1) .
$$

The fundamental class $\kappa_{m+b_{i}-2}$ transgresses to $S q^{b_{i}}\left(\kappa_{m-1}\right)$ in the Serre spectral sequence for this fibration. By $(3.1), \sum_{i} S q^{a_{i}}\left(\kappa_{b_{i}+m-2}\right)$ transgresses to

$$
\sum_{i} S q^{a_{i}} S q^{b_{i}}\left(\kappa_{m-1}\right)=S q^{m}\left(\kappa_{m-1}\right)=0
$$

Thus, the class $\sum_{i} S q^{a_{i}}\left(\kappa_{m+b_{i}-2}\right)$ survives in the spectral sequence and pulls back to an element of $H^{2 m-2}\left(A_{1}\right)$ which we shall call $\phi_{2}$.

Now, consider the diagram below:

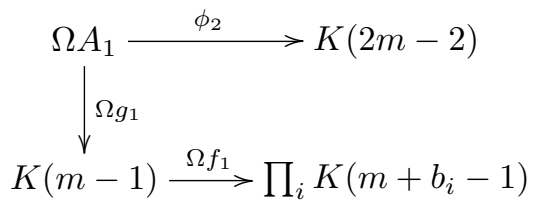

This gives rise to a secondary cohomology operation $\Phi$ which is defined on those elements of $H^{m-1}(X)$ which are annihilated by the $S q^{b_{i}}$. Given such an element $\tau$, we abuse notation, thinking of $\tau$ as a map $\tau: X \rightarrow K(m-1)$ such that $\Omega f_{1} \circ \tau$ is null-homotopic. We may then choose a lifting $\bar{\tau}: X \rightarrow \Omega A_{1}$. Then $\Phi(\tau)$ is defined to be the cohomology class represented by $\phi_{2} \circ \bar{\tau}$, which is independent of the lifting when viewed as an element of $H^{2 m-2}(X) / \oplus_{i} S q^{a_{i}}\left(H^{2 m-2-a_{i}}(X)\right)$. Here 
$\oplus_{i} S q^{a_{i}}\left(H^{2 m-2-a_{i}}(X)\right)$ is the module of indeterminacy, a sum in a graded vector space; any two lifts

$$
\overline{\tau_{1}}, \overline{\tau_{2}}: X \rightarrow \Omega A_{1}
$$

will differ by a composite

$$
X \rightarrow \prod_{i} K\left(m+b_{i}-2\right) \rightarrow \Omega A_{1} \stackrel{\phi_{2}}{\rightarrow} K_{2 m-2}
$$

which represents elements of the aforementioned module of indeterminacy.

Observe the following properties of $\Phi$.

(i) $\Phi$ is natural with respect to maps of spaces.

(ii) For any sphere $S^{l}, \Phi\left(S^{l}\right)$ is zero modulo zero.

(iii) If $\Phi$ is defined on $\Sigma X$, there is a secondary cohomology operation defined on $X$ which we denote by $\sigma \Phi$. The values of $\Phi$ and $\sigma \Phi$ are related by the evident commutative diagram via the cohomology suspension.

(iv) $\Phi$ satisfies the additivity formula, $\Phi(\tau+\gamma)=\Phi(\tau)+\Phi(\gamma)+\tau \gamma$.

Remarks 3.1. We shall prove (iii), but first we offer some remarks on the other observations.

(i) Given $f: Y \rightarrow X$ such that $\Phi$ is defined on $Y$, we see naturality satisfied in the following diagram, where $\bar{\tau} \circ f$ provides the desired lift.

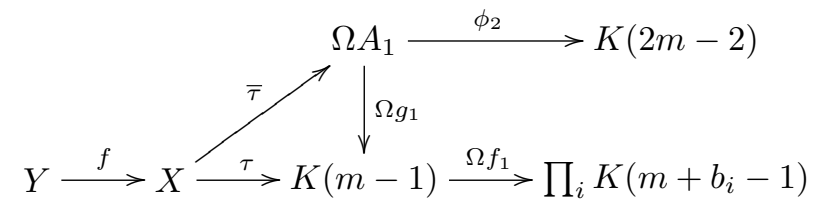

(ii) This follows because of dimensional reasons and the fact that the Steenrod algebra acts trivially on $S^{l}$.

(iv) This is proven in $[\mathbf{B P}]$ using the observation that $\phi_{2}$ is not primitive.

Now, for any space $K$, let $\sigma_{i}: H^{i+1}(K) \rightarrow H^{i}(\Omega K)$ denote the cohomology suspension, a map which commutes with the Steenrod algebra action. We shall use $\sigma_{i}$ to help us prove a notion of stability (iii) for these secondary cohomology operations. In the Serre spectral sequence for the fibration

$$
\prod_{i} \Omega K\left(m+b_{i}-2\right) \stackrel{\Omega^{2} h}{\rightarrow} \Omega^{2} A_{1} \stackrel{\Omega^{2} g_{1}}{\rightarrow} \Omega K(m-1),
$$

the element

$$
\sum_{i} S q^{a_{i}}\left(\sigma_{i+m-3}\left(\kappa_{b_{i}+m-2}\right)\right)
$$

transgresses to

$$
\sum_{i} S q^{a_{i}} S q^{b_{i}}\left(\kappa_{m-2}\right)=S q^{m}\left(\kappa_{m-2}\right)=0 .
$$


Then, $\sum_{i} S q^{a_{i}}\left(\sigma_{i+m-3}\left(\kappa_{b_{i}+m-2}\right)\right)$ survives in the Serre spectral sequence for the looped fibration. This element pulls back to $\sigma_{2 m-3}\left(\phi_{2}\right) \in H^{*}\left(\Omega^{2} A_{1}\right)$. Observe that $\Omega \phi_{2}: \Omega^{2} A_{1} \rightarrow K(2 m-3)$ represents this element.

Then, looping the diagram above gives rise to another secondary cohomology operation, which we shall denote $\sigma \Phi$,

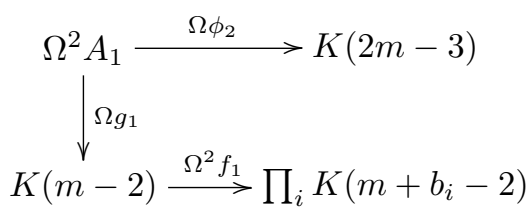

We observe that $\sigma \Phi$ is defined for all $\tau \in H^{m-2}(X)$ such that $S q^{a_{i}}$ annihilates $\tau ; \sigma \Phi$ takes values in $H^{2 m-3}(X) / \oplus_{i} S q^{a_{i}}\left(H^{2 m-3-a_{i}}(X)\right)$.

Now, suppose $\Phi$ is defined on an element $\tau \in H^{m-1}(\Sigma X)$. As above, we abuse notation and think of $\tau$ as a map $\tau: \Sigma X \rightarrow K(m-1)$. So, we have the following diagram where $\phi_{2} \circ \bar{\tau}$ represents $\Phi(\tau)$,

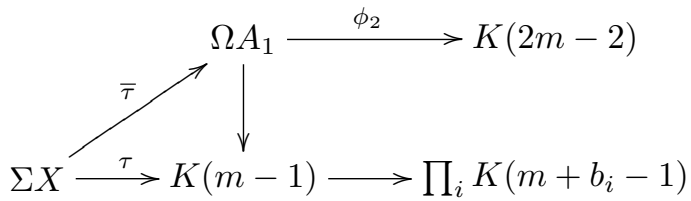

Let $\eta: X \rightarrow \Omega \Sigma X$ be the canonical map. Then $\sigma \Phi$, defined on $\eta \circ \Omega \tau$, is $\Omega \phi_{2} \circ \Omega \bar{\tau} \circ$ $\eta$, as in the diagram below.

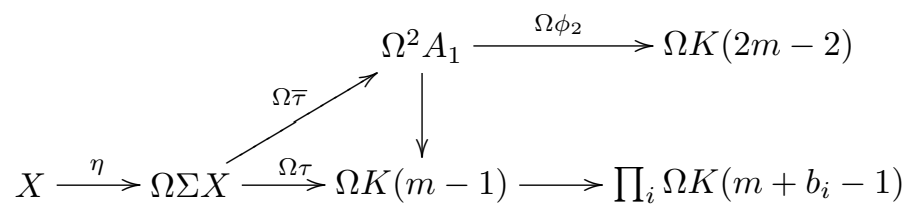

Yet $\phi_{2} \circ \bar{\tau}$ and $\Omega \phi_{2} \circ \Omega \bar{\tau} \circ \eta$ are adjoints of each other. For any space $X$, let $s_{i}$ : $H^{i+1}(\Sigma X) \rightarrow H^{i}(X)$ be the suspension homomorphism. Then, modulo indeterminacy, the following diagram commutes.

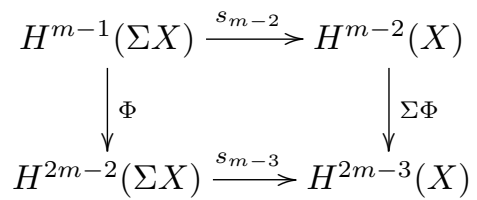

We shall use this notion of stability. Notice that if $\Phi$ is defined on $H^{m-1}(\Sigma X)$, then $\sigma \Phi$ is defined on $H^{m-2}(X)$. 


\subsection{Adams' secondary cohomology operations $\Phi_{i j}$}

In $[\mathbf{A}]$, Adams constructs secondary cohomology operations $\Phi_{i j}$ using minimal resolutions of $\mathbb{Z} / 2 \mathbb{Z}$ over the Steenrod algebra. We briefly look at these operations using the ideas of Brown and Peterson.

Instead of using an equation of the type of $(3.1), \Phi_{i j}$ is based on the relation, discussed in the appendix,

$$
S q^{2^{i}} S q^{2^{j}}+\sum f_{k} S q^{2^{g_{k}}}=0
$$

where $i, j$ are non-negative integers with $i \leqslant j$ and $i+1 \neq j, f_{k}$ is a Steenrod operation, and $g_{k}<j$. As in Section 3.1, we may create a Postnikov system. Given any $m$, we let

$$
f_{1}: K(m) \rightarrow \prod K\left(m+2^{g_{k}}\right) \times K\left(m+2^{j}\right)
$$

be such that the fundamental classes on the right are mapped under $f_{1}^{*}$ to the corresponding Steenrod operation

$$
S q^{2^{g_{k}}}\left(\kappa_{m}\right) \text { or } S q^{2^{j}}\left(\kappa_{m}\right) .
$$

We let $A_{1}$ be the homotopy fiber, denoting the fibration $A_{1} \rightarrow K(m)$ by $g_{1}$. Let

$$
h: \prod_{i} K\left(m+2^{g_{k}}-1\right) \times K\left(m+2^{j}-1\right) \rightarrow A_{1}
$$

be the map of the fiber of $g_{1}$ into $A_{1}$. We notice that the element

$$
\sum f_{k}\left(\kappa_{m+2^{g_{k}}-1}\right)+S q^{2^{i}}\left(\kappa_{m+2^{j}-1}\right)
$$

transgresses to 0 , and thus must pull back to an element $\phi_{i j}$ of $H^{*}\left(A_{1}\right)$ in the Serre spectral sequence for $g_{1}$. Hence, we obtain the following diagram without looping:

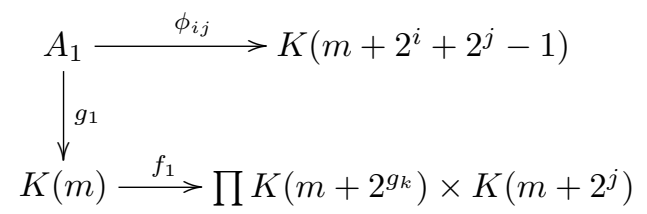

As in Section 3.1, this diagram gives rise to a secondary cohomology operation which we denote $\Phi_{i j}$. The fact that we need not loop the diagram gives rise to a stability property of $\Phi_{i j}$. This also gives rise to the result that $\phi_{i j}$ is primitive, yielding a nice additivity formula for $\Phi_{i j}$. We summarize some properties of this operation:

(i) $\Phi_{i j}$ is natural with respect to maps of spaces.

(ii) For any sphere $S^{l}, \Phi_{i j}\left(S^{l}\right)$ is zero modulo zero.

(iii) $\Phi_{i j}$ is a stable operation: $\Phi_{i j}$ is defined on $X$ if and only if $\Phi_{i j}$ is defined on $\Sigma X$, and the results are related in the obvious way via the cohomology suspension.

(iv) $\Phi_{i j}$ satisfies the additivity formula, $\Phi_{i j}(\tau+\gamma)=\Phi_{i j}(\tau)+\Phi_{i j}(\gamma)$. 
Homology, Homotopy and Applications, vol.9(1), 2007

\subsection{Constructing a tertiary cohomology operation}

This tertiary operation is based on a relation between the stable secondary cohomology operations $\Phi_{i j}$ that Adams develops in $[\mathbf{A}]$. There, he proves the relation,

$$
S q^{2^{r+1}}(\tau)=\sum_{i \leqslant j, i+1 \neq j} a_{i j} \Phi_{i j}(\tau)
$$

for $\tau$ such that $S q^{2^{s}}(\tau)=0$ for $0 \leqslant s \leqslant r$ where $a_{i j}$ are elements of the mod 2 Steenrod algebra. Let $S q^{i}: K\left(2^{r+1}\right) \rightarrow K\left(2^{r+1}+i\right)$ represent the element, $S q^{i}\left(\kappa_{2^{r+1}}\right)$. To create $\Psi$, we look at the map

$$
f_{1}: K\left(2^{r+1}\right) \rightarrow \prod_{0 \leqslant s \leqslant r} K\left(2^{r+1}+2^{s}\right)
$$

such that

$$
\left(f_{1}\right)^{*}\left(\kappa_{2^{r+1}+2^{s}}\right)=S q^{2^{s}}\left(\kappa_{2^{r+1}}\right) .
$$

Denote the homotopy fiber of $f_{1}$ by $A_{1}$ and the map $A_{1} \rightarrow K\left(2^{r+1}\right)$ by $g_{1}$. Applying the Serre spectral sequence in cohomology to the fibration,

$$
\prod_{0 \leqslant s \leqslant r} K\left(2^{r+1}+2^{s}-1\right) \rightarrow A_{1} \rightarrow K\left(2^{r+1}\right)
$$

yields that the fundamental classes of the fiber, $\kappa_{2^{r+1}+2^{s}-1}$, transgress to $S q^{2^{s}}\left(\kappa_{2^{r+1}}\right)$ where $\kappa_{2^{r+1}}$ is the fundamental class of the base. Examining the spectral sequence shows that $\kappa_{2^{r+1}}$ survives to $E_{\infty}$ and corresponds to the element $\left(g_{1}\right)^{*}\left(\kappa_{2^{r+1}}\right)$. Furthermore, there are no elements of $H^{*}\left(A_{1}\right)$ with dimension between $2^{r+1}$ and $2^{r+2}$. For $0 \leqslant s \leqslant r$, it must be the case that $S q^{2^{s}}\left(\left(g_{1}\right)^{*}\left(\kappa_{2^{r+1}}\right)\right)=0$ in $H^{*}\left(A_{1}\right)$. This condition allows us to define $\Phi_{i j}\left(\left(g_{1}\right)^{*}\left(\kappa_{2^{r+1}}\right)\right.$ with zero indeterminacy. Representing these elements is a map $f_{2}: A_{1} \rightarrow \prod_{i \leqslant j, i+1 \neq j} K\left(2^{i}+2^{j}-1+2^{r+1}\right)$ as the degree of $\Phi_{i j}$ is $2^{i}+2^{j}-1$.

We define the homotopy fiber of $f_{2}$ to be $A_{2}$ where $A_{2} \rightarrow A_{1}$ is denoted $g_{2}$. Examining the cohomology Serre spectral sequence of

$$
\prod_{i \leqslant j, i \neq j} K\left(2^{i}+2^{j}-1+2^{r+1}-1\right) \rightarrow A_{2} \rightarrow A_{1}
$$

shows that $v_{i j}$ transgresses to $\Phi_{i j}\left(\left(g_{1}\right)^{*}\left(\kappa_{2^{r+1}}\right)\right)$, where $v_{i j}$ is the fundamental class of $K\left(2^{i}+2^{j}-1+2^{r+1}-1\right)$. Inspired by (3.1) we compute $\sum_{i \leqslant j, i+1 \neq j} a_{i j}\left(v_{i j}\right)$ which transgresses to $S q^{2^{r+1}}\left(\left(g_{1}\right)^{*}\left(\kappa_{2^{r+1}}\right)\right)=\left(\left(g_{1}\right)^{*}\left(\kappa_{2^{r+1}}\right)\right)^{2} \neq 0$.

We loop the maps $f_{1}, f_{2}, g_{1}, g_{2}$ which provides us with a new set of fibrations, in particular,

$$
\prod_{i \leqslant j, i \neq j} K\left(2^{i}+2^{j}-1+2^{r+1}-2\right) \rightarrow \Omega A_{2} \rightarrow \Omega A_{1}
$$


We use $\overline{v_{i j}}$ to denote the fundamental classes in the fiber. Similar to above, we compute $\sum_{i \leqslant j, i+1 \neq j} a_{i j}\left(\overline{v_{i j}}\right)$ which transgresses to $S q^{2^{r+1}}\left(\left(g_{1}\right)^{*}\left(\kappa_{2^{r+1}}-1\right)\right)=0$ for dimensional reasons. Thus, $\sum_{i \leqslant j, i+1 \neq j} a_{i j}\left(\overline{v_{i j}}\right)$ must pull back to an element of $H^{2^{r+2}-2}\left(\Omega A_{2}\right)$ which we denote $\psi_{3}$. We consider the following diagram to better understand how to construct our tertiary operation:

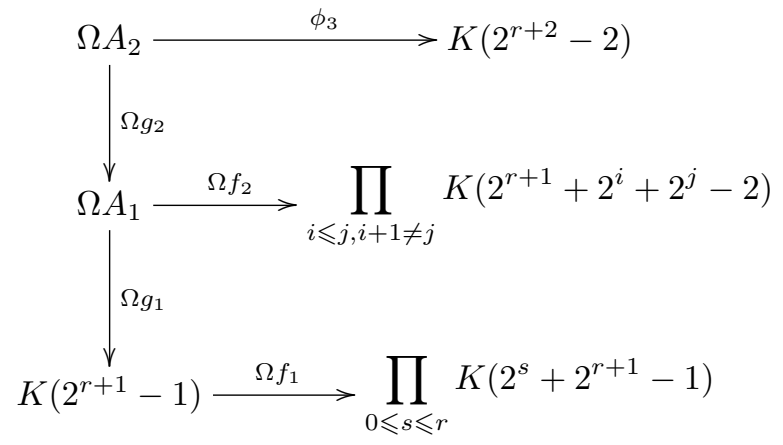

This diagram defines the tertiary operation $\Psi$, which is defined on elements of $H^{2^{r+1}-1}(X)$ with a trivial action under $\Phi_{i j}$.

We list the properties of $\Psi$ followed by an in-depth discussion of the last two properties.

(i) $\Psi$ is natural with respect to maps of spaces.

(ii) For any sphere $S^{l}, \Psi\left(S^{l}\right)$ is zero modulo zero.

(iii) If $\Psi$ is defined on $\Sigma X$, there is a tertiary cohomology operation defined on $X$ which we denote by $\sigma \Psi$. The values of $\Psi$ and $\sigma \Psi$ are related by the evident commutative diagram via the cohomology suspension.

(iv) $\Psi$ satisfies the additivity formula, $\Psi(\tau+\gamma)=\Psi(\tau)+\Psi(\gamma)+\tau \gamma$.

\subsubsection{Stability of $\Psi$}

Similar to our previously developed $\sigma \Phi$, we can construct $\sigma \Psi$ with the property that for spaces $\Sigma X$ in which $\sigma \Psi$ is defined, we have, modulo indeterminacy,

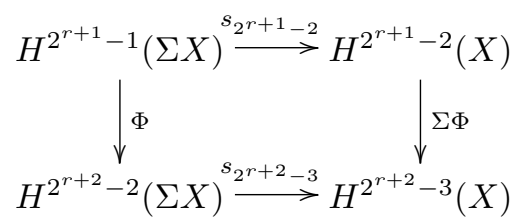

In (3.4), the bottom square corresponds to the construction for $\Phi_{i j}$, which Adams has already proved is a stable operation. Thus, if we loop (3.4) the bottom square still corresponds to the construction of $\Phi_{i j}$. In the Serre spectral sequence of the fibration

$$
\prod_{i \leqslant j, i \neq j} \Omega K\left(2^{i}+2^{j}-1+2^{r+1}-2\right) \rightarrow \Omega^{2} A_{2} \rightarrow \Omega^{2} A_{1}
$$


the element $\sum_{i \leqslant j, i+1 \neq j} a_{i j}\left(\sigma \overline{v_{i j}}\right)$ transgresses to $S q^{2^{r+1}}\left(\left(g_{1}\right)^{*}\left(\sigma \kappa_{2^{r+1}}-1\right)\right)=0$. Thus, $\sum_{i \leqslant j, i+1 \neq j} a_{i j}\left(\sigma \overline{v_{i j}}\right)$ must pull back to $\sigma \psi_{3} \in H^{2^{r+2}-3}\left(\Omega A_{2}, \mathbb{F}_{2}\right)$, which is represented by $\Omega \psi_{3}: \Omega A_{2} \rightarrow K\left(2^{r+2}-3\right)$. The resulting diagram results in a tertiary operation which we denote $\sigma \Psi$.

Suppose $\Psi$ is defined on an element $\tau: \Sigma X \rightarrow K\left(2^{r+1}-1\right)$ so that lifts $\bar{\tau}: X \rightarrow$ $\Omega A_{1}$ and $\overline{\bar{\tau}}: X \rightarrow \Omega A_{2}$ exist. Then $\sigma \Psi$ is defined on the element $\Omega \tau \circ \eta$, where $\eta: X \rightarrow \Omega \Sigma X$ is the canonical map. The relevant lifts $\Omega \bar{\tau} \circ \eta$ and $\Omega \overline{\bar{\tau}} \circ \eta$ exist, and in particular, $\Omega \overline{\bar{\tau}} \circ \eta$ is the adjoint of $\overline{\bar{\tau}}$. It follows that our desired diagram (3.5) exists.

\subsubsection{An additivity formula for $\Psi$}

Similar to the proof of the additivity formula of $\Phi$ in $[\mathbf{B P}]$, we must show that $\phi_{3}$ is not primitive; we use the following result from [W, p. 383],

Lemma 3.2. If $\Omega K$ is $(n-1)$-connected, then the module of primitives of $H^{*}(\Omega K)$ contained in $H^{l}(\Omega K)$ is equal to the image of $\sigma_{l}$ as long as $l \leqslant 3 n-1$.

To apply Lemma 3.2, we compute the connectivity of $\Omega A_{2}$. First, by looking at the long exact sequence of homotopy groups derived from

$$
\prod_{0 \leqslant k \leqslant r} K\left(2^{k}+2^{r+1}-1\right) \rightarrow A_{1} \rightarrow K\left(2^{r+1}\right)
$$

we see the connectivity of $A_{1}$ is $2^{r+1}-1$. Examining the long exact sequence of homotopy groups for $\prod_{i \leqslant j, i+1 \neq j} K\left(2^{r+1}+2^{i}+2^{j}-1\right) \rightarrow A_{2} \rightarrow A_{1}$ gives that the connectivity of $A_{2}$ is also $2^{r+1}-1$; thus, the connectivity of $\Omega A_{2}$ is $2^{r+1}-2$. Since $r>3$

$$
2^{r+2}-2<3\left(2^{r+1}\right)-1
$$

By Lemma 3.2 it follows that $\phi_{3}$ is primitive if and only if $\phi_{3}=\sigma_{2^{r+2}-2}(\psi)$ for some $\psi \in H^{2^{r+2}-1}\left(A_{2}\right)$. We assume $\psi$ exists and show that

$$
h^{*}(\psi)=\sum_{i \leqslant j, i+1 \neq j} a_{i j} v_{i j}
$$

Then, $h^{*}(\psi)$ transgresses to zero in the spectral sequence induced by the fibration $\prod_{i \leqslant j, i+1 \neq j} K\left(2^{r+1}+2^{i}+2^{j}-1\right) \stackrel{h}{\rightarrow} A_{2} \rightarrow A_{1}$. However, we have seen $\sum_{i \leqslant j, i+1 \neq j} a_{i j} v_{i j}$ transgresses to $S q^{2^{r+1}}\left(\left(g_{1}\right)^{*}\left(\kappa_{2^{r+1}}\right)\right)=\left(\left(g_{1}\right)^{*}\left(\kappa_{2^{r+1}}\right)\right)^{2} \neq 0$. This gives us the desired contradiction, and hence, $\phi_{3}$ is not primitive. 
Homology, Homotopy and Applications, vol. 9(1), 2007

We assume $\psi$ exists. Then,

$$
\begin{aligned}
\sigma_{2^{r+2}-2}\left[(h)^{*}(\psi)\right] & =(\Omega h)^{*}\left(\sigma_{2^{r+2}-1}(\psi)\right) \\
& =(\Omega h)^{*}\left(\phi_{3}\right) \\
& =\sum_{i \leqslant j, i+1 \neq j} a_{i j}\left(\overline{v_{i j}}\right) \\
& =\sigma_{2^{r+2}-2}\left[\sum_{i \leqslant j, i+1 \neq j} a_{i j}\left(v_{i j}\right)\right] .
\end{aligned}
$$

We show $\sum_{i \leqslant j, i+1 \neq j} a_{i j}\left(\overline{v_{i j}}\right)$ is the only pre-image of $\sigma_{2^{r+2}-2}\left[\sum_{i \leqslant j, i+1 \neq j} a_{i j}\left(v_{i j}\right)\right]$ under

$$
\begin{gathered}
\sigma_{2^{r+2}-2}: H^{2^{r+2}-1}\left(\prod_{i \leqslant j, i+1 \neq j} K\left(2^{i}+2^{j}+2^{r+1}-2\right)\right) \rightarrow \\
H^{2^{r+2}-2}\left(\prod_{i \leqslant j, i+1 \neq j} K\left(2^{i}+2^{j}+2^{r+1}-3\right)\right) .
\end{gathered}
$$

The Hurewicz dimension of the Eilenberg MacLane spaces in the product,

$$
\prod_{i \leqslant j, i+1 \neq j} K\left(2^{i}+2^{j}+2^{r+1}-2\right)
$$

is at least $2^{r+1}$, when $i=j=0$. Then, $H^{2^{r+2}-1}\left(\prod_{i \leqslant j, i+1 \neq j} K\left(2^{i}+2^{j}+2^{r+1}-2\right)\right)$ contains no decomposables, and so $\sigma_{2^{r+2}-2}$ is a monomorphism. It must be the case that $h^{*}(\psi)=\sum_{i \leqslant j, i+1 \neq j} a_{i j} v_{i j}$, and we obtain the desired contradiction. We have established that $\Omega A_{2}$ is $\left(2^{r+1}-2\right)$-connected. By the Künneth Theorem,

$$
\begin{gathered}
H^{2^{r+2}-2}\left(\Omega A_{2} \times \Omega A_{2}\right)= \\
H^{2^{r+2}-2}\left(\Omega A_{2}\right) \oplus H^{2^{r+1}-1}\left(\Omega A_{2}\right) \otimes H^{2^{r+1}-1}\left(\Omega A_{2}\right) \oplus H^{2^{r+2}-2}\left(\Omega A_{2}\right) .
\end{gathered}
$$

Recall that we have a fibration, $\prod_{i \leqslant j, i \neq j} K\left(2^{r+1}+2^{i}+2^{j}-3\right) \stackrel{\Omega^{2} f_{2}}{\rightarrow} \Omega A_{2} \stackrel{\Omega g_{2}}{\rightarrow} \Omega A_{1}$.

Examining the Serre spectral sequence applied to this fibration, we see that

$$
\left(\Omega g_{2}\right)^{*}\left(\Omega g_{1}\right)^{*}\left(\kappa_{2^{r+1}-1}\right) \otimes\left(\Omega g_{2}\right)^{*}\left(\Omega g_{1}\right)^{*}\left(\kappa_{2^{r+1}-1}\right)
$$

is the only non-trivial element of $H^{2^{r+1}-1}\left(\Omega A_{2}\right) \otimes H^{2^{r+1}-1}\left(\Omega A_{2}\right)$. Define $v: \Omega A_{2} \times$ $\Omega A_{2} \rightarrow \Omega A_{2}$ to be the loop multiplication map. Thus, since $\phi_{3}$ is not primitive,

$$
v^{*}\left(\phi_{3}\right)=\phi_{3} \otimes 1+\left(\Omega g_{2}\right)^{*}\left(\Omega g_{1}\right)^{*}\left(\kappa_{2^{r+1}-1}\right) \otimes\left(\Omega g_{2}\right)^{*}\left(\Omega g_{1}\right)^{*}\left(\kappa_{2^{r+1}-1}\right)+1 \otimes \phi_{3} .
$$

Let $X$ be a space such that there exist $\tau, \gamma \in H^{2^{r+1}-1}\left(X, \mathbb{F}_{2}\right)$ such that $\Psi$ is defined for $\tau$ and $\gamma$. Consider the maps that represent these elements

$$
\tau: X \rightarrow K\left(2^{r+1}-1\right)
$$

and 


$$
\gamma: X \rightarrow K\left(2^{r+1}-1\right)
$$

By assumption, it must be the case that $S q^{2^{k}}$ acts trivally on $\tau$ and $\gamma$ for $0 \leqslant k \leqslant r$, so we may find lifts $\bar{\tau}, \bar{\gamma}: X \rightarrow \Omega A_{1}$. Again, by assumption, Adams' $\Phi_{i j}$ are defined and zero on these elements, so we have lifts $\overline{\bar{\tau}}, \overline{\bar{\gamma}}: X \rightarrow \Omega A_{2}$. Let $\triangle: X \rightarrow X \times X$ be the diagonal map, and $\mu: \Omega K\left(2^{r+1}-1\right) \times \Omega K\left(2^{r+1}-1\right) \rightarrow \Omega K\left(2^{r+1}-1\right)$ be the multiplication map. Now,

$$
\begin{aligned}
\Omega g_{1} \circ \Omega g_{2} \circ v \circ(\overline{\bar{\tau}} \times \overline{\bar{\gamma}}) \circ \triangle & =\mu \circ(\tau \times \gamma) \circ \triangle \\
& =\tau+\gamma .
\end{aligned}
$$

So, $v \circ(\overline{\bar{\tau}} \times \overline{\bar{\gamma}}) \circ \triangle$ is a lift of $\tau+\gamma$ under the map $\Omega g_{1} \circ \Omega g_{2}$ as well as a lift of $v \circ(\bar{\tau}+\bar{\gamma}) \circ \triangle$ through $\Omega g_{2}$. The map $v \circ(\bar{\tau}+\bar{\gamma}) \circ \triangle$, itself, is a lift of $\tau+\gamma$ under $\Omega g_{1}$. So modulo the indeterminacy of $\Psi$, we have

$$
\begin{aligned}
\Psi(\tau+\gamma) & =(v \circ(\overline{\bar{\tau}} \times \overline{\bar{\gamma}}) \circ \triangle)^{*} \phi_{3} \\
& =(\overline{\bar{\tau}} \times \overline{\bar{\gamma}} \circ \triangle)^{*}\left(\phi_{3} \otimes 1+\xi \otimes \xi+1 \otimes \phi_{3}\right) \\
& =\Psi(\tau)+\Psi(\gamma)+\tau \gamma,
\end{aligned}
$$

where $\xi=\left(\Omega g_{2}\right)^{*}\left(\Omega g_{1}\right)^{*}\left(\kappa_{2^{r+1}-1}\right)$.

\section{Showing $\Omega S^{n}$ is minimal atomic}

We show for positive integers $n>1$ that $\Omega S^{n}$ is minimal atomic for $n \neq 2,4,8$ by means of secondary and tertiary cohomology operations. These arguments will show in detail how certain cohomology classes are tied together. We first determine the spherical candidates of $\Omega S^{n}$ and show that above the Hurewicz dimension, these candidates are in the target of a higher order cohomology operation.

\subsection{Spherical candidates of $\Omega S^{n}$}

To determine which elements of $H_{*}\left(\Omega S^{n}\right)$ are primitive and annihilated by the Steenrod algebra, we note that the Steenrod operations on $\Omega S^{n}$ are trivial. Discussed in [S, p. 85], the James construction applied to $S^{n-1}, J\left(S^{n-1}\right)$, is homotopy equivalent to $\Omega \Sigma S^{n-1}=\Omega S^{n}$. Using the splitting property of the suspension of the James construction, we have $\Sigma \Omega \Sigma S^{n-1}=\bigvee_{k=1}^{\infty} \Sigma S^{k(n-1)}$. Since the Steenrod operations on $\Sigma S^{k(n-1)}$ are trivial, we deduce that the Steenrod operations are trivial on $\Sigma \Omega S^{n}$ and thus, on $\Omega S^{n}$. So, all of the primitive elements above the Hurewicz homomorphism could conceivably lie in the image of the mod 2 Hurewicz homomorphism.

Computations with the Serre spectral sequence on the path fibration $\Omega S^{n} \rightarrow$ $P S^{n} \rightarrow S^{n}$ allow us to conclude that $H^{*}\left(\Omega S^{n}\right)=\Gamma\left[\alpha_{n-1}\right]$ as a Hopf algebra. Alternatively, as an algebra, $H^{*}\left(\Omega S^{n}\right)=\bigotimes_{k \geqslant 0} P\left[\gamma_{2^{k}}\left(\alpha_{n-1}\right)\right] /\left(\gamma_{2^{k}}\left(\alpha_{n-1}\right)\right)^{2}$; the binomial coefficients appearing in the multiplication of the divided polynomial algebra reduce 
to give $\Gamma\left[\alpha_{n-1}\right]=\bigotimes_{k \geqslant 0} P\left[\gamma_{2^{k}}\left(\alpha_{n-1}\right)\right] /\left(\gamma_{2^{k}}\left(\alpha_{n-1}\right)\right)^{2}$. Also, observe that only one element exists in dimension $2^{k}(n-1)$, namely $\gamma_{2^{k}}\left(\alpha_{n-1}\right)$. If there were another element of dimension $2^{k}(n-1)$, such an element would be a product of distinct generators $\gamma_{2^{i}}\left(\alpha_{n-1}\right)$ with $i<k$. However, the largest degree achieved by elements of this form is $2^{k}(n-1)-1=\left|\prod_{i=0}^{k-1} \gamma_{2^{i}}\left(\alpha_{n-1}\right)\right|$. We will use this fact later. Let $a_{k} \in H_{*}\left(\Omega S^{n}\right)$ be the dual element to $\gamma_{2^{k}}\left(\alpha_{n-1}\right)$.

4.2. $n \neq 2^{r+1}$

When $n \neq 2^{r+1}, S q^{n}$ has a factorization in the Steenrod algebra. This factorization, along with factorizations of $S q^{2^{k}(n-1)}$ for $k \geqslant 1$, will be utilized to construct Brown-Peterson secondary cohomology operations necessary to show $\Omega S^{n}$ is minimal atomic. Now, to apply these operations to $\Omega S^{n}$ we must specify examples of our secondary cohomology operations $\Phi$. Set $n=2^{r}+a$ where $2^{r}$ is the largest power of 2 which appears in the binary representation of $n$. For now, we suppose that $n$ is not a power of 2 so that $0<a<2^{r}$. By taking the binary representations of $2^{r}-1$ and $a$, we see that $\left(\begin{array}{c}2^{r}-1 \\ a\end{array}\right)=1 \bmod 2$ : Recall the calculation

$$
\left(\begin{array}{l}
i \\
j
\end{array}\right)=\prod\left(\begin{array}{l}
i_{k} \\
j_{k}
\end{array}\right) \bmod 2
$$

where $i_{k}$ is the $k$ th term in the binary representation of $i$ and similarly for $j_{k}$. The binary representation of $2^{r}-1$ consists of $r$ uninterrupted 1's; the binary representation of $a$ is at most $r$ digits long. Applying the result above yields $\left(\begin{array}{c}2^{r}-1 \\ a\end{array}\right)=1$. Using the Adem relations, where the binomial coefficients are taken mod 2, gives

$$
S q^{a} S q^{2^{r}}=S q^{n}+\sum_{c>0}\left(\begin{array}{c}
2^{r}-c-1 \\
a-2 c
\end{array}\right) S q^{n-c} S q^{c} .
$$

Then,

$$
S q^{n}=S q^{a} S q^{2^{r}}+\sum_{c \in S}\left(\begin{array}{c}
2^{r}-c-1 \\
a-2 c
\end{array}\right) S q^{n-c} S q^{c}
$$

where $S=\left\{c \mid 2 c \leqslant a\right.$ and $\left.\left(\begin{array}{c}2^{r}-c-1 \\ a-2 c\end{array}\right) \neq 0 \bmod 2\right\}$.

As we have seen, this relation gives rise to a secondary cohomology operation $\Phi_{0}$ which acts on elements of $H^{n-1}\left(\Omega S^{n}\right)$ that vanish under $S q^{n}$ and $S q^{c}$ for $c \in S$ above, and takes values in $H^{2 n-2}\left(\Omega S^{n}\right) / S q^{a} H^{2 n-2-a}\left(\Omega S^{n}\right) \oplus \bigoplus_{c \in S} H^{2 n-2-c}\left(\Omega S^{n}\right)$. Furthermore, we know that $\Phi_{0}(\tau+\gamma)=\Phi_{0}(\tau)+\Phi_{0}(\gamma)+\tau \gamma$ where $\Phi_{0}$ is defined on $\tau$ and $\gamma$.

We have the loop multiplication map $\omega: \Omega S^{n} \times \Omega S^{n} \rightarrow \Omega S^{n}$. We note that since the Steenrod operations on $H^{*}\left(\Omega S^{n}\right)$ are trivial, $\Phi_{0}\left(\gamma_{1}\left(\alpha_{n-1}\right)\right)$ is defined with zero 
indeterminacy. Then the Steenrod operations on $H^{*}\left(\Omega S^{n} \times \Omega S^{n}\right)$ are trivial, so $\Phi_{0}$ is defined on $H^{*}\left(\Omega S^{n} \times \Omega S^{n}\right)$ with zero indeterminacy. Then,

$$
\begin{aligned}
\omega^{*} \Phi_{0}\left(\gamma_{1}\left(\alpha_{n-1}\right)\right)= & \Phi_{0}\left(\omega^{*}\left(\gamma_{1}\left(\alpha_{n-1}\right)\right)\right) \\
= & \Phi_{0}\left(\gamma_{1}\left(\alpha_{n-1}\right) \otimes 1+1 \otimes \gamma_{1}\left(\alpha_{n-1}\right)\right) \\
= & \Phi_{0}\left(\gamma_{1}\left(\alpha_{n-1}\right)\right) \otimes 1+1 \otimes \Phi_{0}\left(\gamma_{1}\left(\alpha_{n-1}\right)\right)+ \\
& \gamma_{1}\left(\alpha_{n-1}\right) \otimes \gamma_{1}\left(\alpha_{n-1}\right) .
\end{aligned}
$$

Since $\gamma_{1}\left(\alpha_{n-1}\right) \otimes \gamma_{1}\left(\alpha_{n-1}\right) \neq 0$, we have that $\omega^{*}\left(\Phi_{0}\left(\gamma_{1}\left(\alpha_{n-1}\right)\right)\right.$ is non-zero. Then, $\Phi_{0}\left(\gamma_{1}\left(\alpha_{n-1}\right)\right)$ is non-zero on $H^{(n-1)}\left(\Omega S^{n}\right)$. For dimensional reasons, it must then be the case that

$$
\Phi_{0}\left(\gamma_{1}\left(\alpha_{n-1}\right)\right)=\gamma_{2}\left(\alpha_{n-1}\right) \text {. }
$$

Following Section 2.3, it must be the case that $a_{1}$ is not spherical.

We offer here a proof strictly using secondary cohomology operations to show that the remaining $a_{k+1}$ (with $k>0$ ) are also not spherical. In addition to showing that some elements of $H^{*}\left(\Omega S^{n}\right)$ are related by secondary cohomology operations the results of this argument will be useful when we look at $\Omega^{2} S^{n}$.

Consider the Adem relation, where $k \geqslant 1$ :

$$
S q^{1} S q^{2^{k}(n-1)}=\left(\begin{array}{c}
2^{k}(n-1)-1 \\
1
\end{array}\right) S q^{2^{k}(n-1)+1}=S q^{2^{k}(n-1)+1} .
$$

This yields,

$$
S q^{2^{k}(n-1)+1}=S q^{1} S q^{2^{k}(n-1)} .
$$

Let us call the secondary cohomology operations which stem from this relation $\Phi_{k}$. So, $\Phi_{k}$ will act on elements of $H^{2^{k}(n-1)}(X)$ which vanish under $S q^{2^{k}(n-1)}$, and will take values in $H^{2^{k+1}(n-1)}(X) / S q^{1}\left(H^{2^{k+1}(n-1)}(X)\right)$. Since the Steenrod operations act trivially on $\Omega S^{n}, \Phi_{k}$ is defined on $H^{2^{k}(n-1)}\left(\Omega S^{n}\right)$ with no indeterminacy. To show that $\Phi_{k}$ is non-zero, we use the following result from $[\mathbf{Z}]$.

Lemma 4.1. Let $X$ and $Y$ be $C W$ complexes. Suppose $z=\sum_{i} \tau_{i} \otimes \gamma_{i} \in H^{m}(X \times$ $Y)$ is in the domain of $\Phi_{k}$ with $\left|\tau_{i}\right|,\left|\gamma_{i}\right|>0$. Let $\rho(\tau)$ and $\rho(\gamma)$ be the algebras over the Steenrod algebra generated by the $\tau_{i}$ 's and $\gamma_{i}$ 's respectively. Then,

$$
\Phi_{k}(z) \cap\left[\rho(\tau) \otimes H^{*}(Y)+H^{*}(X) \otimes \rho(\gamma)\right] \neq \emptyset .
$$

In order to apply this result, we study the action of $\Phi_{k}$ on $\Omega S^{n} \times \Omega S^{n}$. Again, the Steenrod operations act trivially on $\Omega S^{n} \times \Omega S^{n}$; so, $\Phi_{k}$ is defined with zero indeterminacy on $H^{2^{k}(n-1)}\left(\Omega S^{n} \times \Omega S^{n}\right)$. Recall that

$$
\omega\left(\gamma_{2^{k}}\left(\alpha_{n-1}\right)\right)=\left[1 \otimes \gamma_{2^{k}}\left(\alpha_{n-1}\right)\right]+\left[\gamma_{2^{k}}\left(\alpha_{n-1}\right) \otimes 1\right]+\left[\sum_{i} \tau_{i} \otimes \gamma_{i}\right]
$$

where $2^{k}(n-1)>\left|\tau_{i}\right|,\left|\gamma_{i}\right|>0$. Let $z, \rho(\tau), \rho(\gamma)$ be as in the statement of Lemma 4.1. We observe that $\gamma_{2^{k}}\left(\alpha_{n-1}\right) \notin \rho(\tau), \rho(\gamma)$ since $\gamma_{2^{k}}\left(\alpha_{n-1}\right)$ is an indecomposable element of $H^{*}\left(\Omega S^{n}\right)$. Then, 
Homology, Homotopy and Applications, vol. 9(1), 2007

$$
\gamma_{2^{k}}\left(\alpha_{n-1}\right) \otimes \gamma_{2^{k}}\left(\alpha_{n-1}\right) \notin\left[\rho(\tau) \otimes H^{*}\left(\Omega S^{n}\right)+H^{*}\left(\Omega S^{n}\right) \otimes \rho(\gamma)\right] .
$$

Certainly $z$ lies in the domain of $\Phi_{k}$. Because there is no indeterminacy, $\Phi_{k}(z)$ is a singleton set, rather than a coset. So, applying Lemma 4.1, we have

$$
\Phi_{k}(z) \in\left[\rho(\tau) \otimes H^{*}\left(\Omega S^{n}\right)+H^{*}\left(\Omega S^{n}\right) \otimes \rho(\gamma)\right] .
$$

So, $\gamma_{2^{k}}\left(\alpha_{n-1}\right) \otimes \gamma_{2^{k}}\left(\alpha_{n-1}\right)$ is not a summand of $\Phi_{k}(z)$. Further,

$$
\gamma_{2^{k}}\left(\alpha_{n-1}\right) \otimes \gamma_{2^{k}}\left(\alpha_{n-1}\right)
$$

is not a summand of

$$
\left(\gamma_{2^{k}}\left(\alpha_{n-1}\right) \otimes 1+1 \otimes \gamma_{2^{k}}\left(\alpha_{n-1}\right)\right) z
$$

since $\gamma_{2^{k}}\left(\alpha_{n-1}\right)$ is indecomposable and $z=\sum_{i} \tau_{i} \otimes \gamma_{i}$ with $\left|\tau_{i}\right|,\left|\gamma_{i}\right|>0$. We evaluate

$$
\begin{aligned}
\omega^{*} \Phi_{k}\left(\gamma_{2^{k}}\left(\alpha_{n-1}\right)\right) & =\Phi_{k}\left(\omega^{*}\left(\gamma_{2^{k}}\left(\alpha_{n-1}\right)\right)\right) \\
& =\Phi_{k}\left(\gamma_{2^{k}}\left(\alpha_{n-1}\right) \otimes 1+1 \otimes \gamma_{2^{k}}\left(\alpha_{n-1}\right)+z\right) .
\end{aligned}
$$

By utilizing the the Additivity Formula, this expression evaluates to

$$
\begin{gathered}
\Phi_{k}\left(\gamma_{2^{k}}\left(\alpha_{n-1}\right)\right) \otimes 1+ \\
\left(\gamma_{2^{k}}\left(\alpha_{n-1}\right) \otimes 1+1 \otimes \Phi_{k}\left(\gamma_{2^{k}}\left(\alpha_{n-1}\right)\right)+\gamma_{2^{k}}\left(\alpha_{n-1}\right) \otimes \gamma_{2^{k}}\left(\alpha_{n-1}\right)\right) z
\end{gathered}
$$

From our observations above, we note that $\gamma_{2^{k}}\left(\alpha_{n-1}\right) \otimes \gamma_{2^{k}}\left(\alpha_{n-1}\right)$ does not cancel out. Hence,

$$
\omega^{*} \Phi_{k}\left(\gamma_{2^{k}}\left(\alpha_{n-1}\right)\right) \neq 0
$$

Then, $\Phi_{k}\left(\gamma_{2^{k}}\left(\alpha_{n-1}\right)\right) \neq 0$ and for dimensional reasons, it must be the case that

$$
\Phi_{k}\left(\gamma_{2^{k}}\left(\alpha_{n-1}\right)\right)=\gamma_{2^{k+1}}\left(\alpha_{n-1}\right) .
$$

As demonstrated in Section 2.3, we may conclude that $a_{k+1}$ is not spherical. Thus, we have shown for all $k \geqslant 0, a_{k+1}$ is not spherical. So, $\Omega S^{n}$ is minimal atomic for $n$ not a power of 2 .

\section{3. $n=2^{r+1}$ for $r \geqslant 3$}

Our method is similar to that above. To show $a_{k+1}$ is not spherical for each $k \geqslant 0$, we exhibit a cohomology operation whose image hits $\gamma_{2^{k+1}}\left(\alpha_{n-1}\right)$. Happily, our previous argument goes through in the case $k \geqslant 1$. The previously established $\Phi_{k}$ have the property that $\Phi_{k}\left(\gamma_{2^{k}}\left(\alpha_{n-1}\right)\right)=\gamma_{2^{k+1}}\left(\alpha_{n-1}\right)$. So, we still must show that $a_{1}$ is not spherical. To show that $a_{1}$ is not spherical, we show that the tertiary operation $\Psi$, constructed in Subsection 3.3 has the property that $\Psi\left(\gamma_{1}\left(\alpha_{n-1}\right)\right)=\gamma_{2}\left(\alpha_{n-1}\right)$.

Let us evaluate $\Psi$ on the element, $\gamma_{1}\left(\alpha_{n-1}\right) \in H^{n-1}\left(\Omega S^{n}\right)$. To show that $\Psi$ acts on $\gamma_{1}\left(\alpha_{n-1}\right)$, we first recall that the Steenrod operations act trivally on $\Omega S^{n}$. Thus, if $\gamma_{1}\left(\alpha_{n-1}\right)$ is represented by a map $\tau: \Omega S^{n} \rightarrow K\left(2^{r+1}-1\right)$, we have a lift $\bar{\tau}: \Omega S^{n} \rightarrow \Omega A_{1}$. The composite $\Omega f_{2} \circ \bar{\tau}$ represents the product of cohomology classes $\prod_{i \leqslant j, i+1 \neq j} \Phi_{i j}(\tau)$. Recall that $\Sigma \Omega S^{n}$ is homotopy equivalent to $\bigvee_{i=1}^{\infty} S^{i(n-1)}$. We know 
that $\Phi_{i j}$ is trivial on $S^{i(n-1)}$ for $i \geqslant 1$, so $\Phi_{i j}$ is trivial on $\Sigma \Omega S^{n}$. Because $\Phi_{i j}$ is a stable cohomology operation, we have that $\Phi_{i j}$ is zero on $\Omega S^{n}$. Thus, $\Phi_{i j}(\tau)$ is zero with zero indeterminacy, and we have the essential lift $\overline{\bar{\tau}}: \Omega S^{n} \rightarrow \Omega A_{2}$. That is, $\Psi(\tau)$ is defined.

Appealing to Section 4.2 , with $\Psi$ replaced by $\Phi_{0}$, we see that $\Psi\left(\gamma_{1}\left(\alpha_{n-1}\right)\right)$ is non-zero. For dimensional reasons, we have

$$
\Psi\left(\gamma_{1}\left(\alpha_{n-1}\right)\right)=\gamma_{2}\left(\alpha_{n-1}\right)
$$

Thus, as outlined in Section 2.3, $a_{1}$, the dual element of $\gamma_{2}\left(\alpha_{n-1}\right)$, cannot be spherical.

We have shown that no spherical elements of $\Omega S^{n}$ exist above the Hurewicz dimension. Thus, $\Omega S^{n}$ is minimal atomic for $n=2^{r+1}$ where $r \geqslant 3$. In summary, we have shown that $\Omega S^{n}$ is minimal atomic for $n$ where $n \neq 2^{r+1}$ for $r<3$.

\section{Showing $S^{n}\left\{2^{r}\right\}$ for $r>1$ is minimal atomic}

We show that $S^{n}\left\{2^{r}\right\}$ is minimal atomic when $n$ is not a power of 2 . When $n$ is a power of 2 with $n \neq 1,2,4,8$, we are not able to prove $S^{n}\left\{2^{r}\right\}$ is minimal atomic, but we carry out an examination using higher order cohomology operations and illustrate what is lacking to carry out a complete proof. Here, our spaces are automatically 2-local by inspection of homotopy groups.

Recall that $S^{n}\left\{2^{r}\right\}$ is defined to be the homotopy fiber of the degree $2^{r}$ map $f: S^{n} \rightarrow S^{n}$. A long exact sequence of homotopy groups arises:

$$
\cdots \rightarrow \pi_{k+1}\left(S^{n}\right) \stackrel{2^{r}}{\rightarrow} \pi_{k+1}\left(S^{n}\right) \rightarrow \pi_{k}\left(S^{n}\left\{2^{r}\right\}\right) \rightarrow \pi_{k}\left(S^{n}\right) \stackrel{2^{r}}{\rightarrow} \pi_{k}\left(S^{n}\right) \rightarrow \cdots
$$

By examining the cases when $k \leqslant n-1$, we see that $S^{n}\left\{2^{r}\right\}$ is $(n-2)$ connected with $\pi_{(n-1)}\left(S^{n}\left\{2^{r}\right\}\right)=\mathbb{Z} / 2^{r} \mathbb{Z}$. This shows that $S^{n}\left\{2^{r}\right\}$ is a Hurewicz complex. We see that $\pi_{n}\left(S^{n}\left\{2^{r}\right\}\right)=\pi_{n+1}\left(S^{n}\right) / 2^{r} \pi_{n+1}\left(S^{n}\right)$ for $k=n$. Since $\pi_{n+1}\left(S^{n}\right)=\mathbb{Z} / 2 \mathbb{Z}$, we have that $\pi_{n}\left(S^{n}\left\{2^{r}\right\}\right)=\mathbb{Z} / 2 \mathbb{Z}$. Now $\pi_{4 n-1}\left(S^{2 n}\right)$ is a direct sum of $\mathbb{Z}$ and a finite group. Otherwise, $\pi_{q}\left(S^{n}\right)$ is finite if $q>n$. These facts allow us to deduce that $\pi_{k}\left(S^{n}\left\{2^{r}\right\}\right)$ is composed strictly of 2-groups.

\subsection{Spherical candidates of $S^{n}\left\{2^{r}\right\}$}

Computations with the Serre spectral sequence on the induced fibration $\Omega S^{n} \rightarrow$ $S^{n}\left\{2^{r}\right\} \rightarrow S^{n}$ allow us to conclude that

$$
H^{*}\left(S^{n}\left\{2^{r}\right\}, \mathbb{F}_{2}\right)=P\left[\gamma_{2^{k}}\left(\alpha_{n-1}\right)\right] /\left(\gamma_{2^{k}}\left(\alpha_{n-1}\right)\right)^{2} \otimes E\left[\beta_{n}\right]
$$

with trivial Steenrod operation action. So, the primitive elements of $S^{n}\left\{2^{r}\right\}$ are the duals of the elements $\gamma_{2^{k}}\left(\alpha_{n-1}\right)$ and $\beta_{n}$ which we shall label $a_{k}$ and $b_{n}$. Since the image of the Hurewicz homomorphism is contained in the primitive elements of $S^{n}\left\{2^{r}\right\}$, we must show for $k \geqslant 0$ that $a_{k+1}$ and $b_{n}$ are not spherical. First, we examine $b_{n}$.

If $b_{n}$ is spherical, there exists a non-trivial map $j: S^{n} \rightarrow S^{n}\left\{2^{r}\right\}$ which induces an isomorphism in mod 2 homology. By naturality, we have the following diagram: 
Homology, Homotopy and Applications, vol. 9(1), 2007

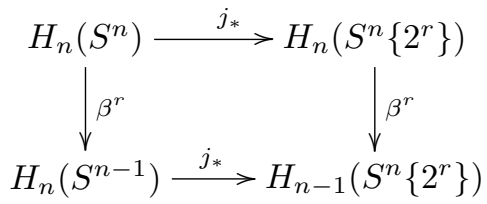

where $\beta^{r}$ is the $r$ th Bockstein operator. Now, $\beta^{r} \circ j_{*}$ hits the element $\gamma_{1}\left(\alpha_{n-1}\right)$ while $j_{*} \circ \beta^{r}$ is trivial. We have a contradiction, and so $b_{n}$ cannot be spherical.

5.2. $n \neq 2^{r+1}$

Now, if $n$ is not a power of 2, our proof of the minimal atomicity of $\Omega S^{n}$ applies exactly with $\Omega S^{n}$ replaced by $S^{n}\left\{2^{r}\right\}$. Since the Steenrod operations on $S^{n}\left\{2^{r}\right\}$ are trivial, we may use the same secondary cohomology operations, $\Phi_{k}$ for $k \geqslant 0$ to show that the $a_{k+1}$ are not spherical.

\section{3. $n=2^{r+1}$ for $r \geqslant 3$}

If $n=2^{r}$ for some $r$ with $n \neq 1,2,4,8$ we will see that modelling the proof for $\Omega S^{n}$ has a stumbling block. As before, for $k \geqslant 1$, the secondary cohomology operations $\Phi_{k}$ show that $a_{k+1}$ is not spherical. So, we still have some work to do to show that $a_{1}$ is not spherical. Fred Cohen has also pointed out that an analogous James-Hopf map $S^{n}\left\{2^{r}\right\} \rightarrow S^{2 n+1}\left\{2^{r}\right\}$ exists which can be used inductively to show that the minimal atomicity of $S^{n}\left\{2^{r}\right\}$ reduces to the problem of whether $a_{1}$ is spherical.

If we try to apply our tertiary operation, $\Psi$, to $\gamma_{1}\left(\alpha_{n-1}\right)$ we must check that the $\Phi_{i j}$ are zero on $\gamma_{1}\left(\alpha_{n-1}\right)$. We observe that the $\Phi_{i j}$ are defined on $S^{n}\left\{2^{r}\right\}$ since the Steenrod operations act trivially on $S^{n}\left\{2^{r}\right\}$. Recall that $i, j \leqslant r-1$ and

$$
\left|\Phi_{i j}\left(\gamma_{1}\left(\alpha_{n-1}\right)\right)\right|=2^{i}+2^{j}+2^{r}-2>2^{r}-1 \text {. }
$$

The largest value for $2^{i}+2^{j}+2^{r}-2$ is $2^{r+1}-2=\left|\gamma_{2}\left(\alpha_{n-1}\right)\right|$. Based solely on degree considerations, then, it is possible that $\Phi_{r-1, r-1}\left(\gamma_{1}\left(\alpha_{n-1}\right)\right)$ is not zero since it might take on the value $\gamma_{2}\left(\alpha_{n-1}\right)$. Other potential values of $\Phi_{i j}\left(\gamma_{1}\left(\alpha_{n-1}\right)\right)$ are $\beta_{n}$ and $\beta_{n} \gamma_{1}\left(\alpha_{n-1}\right)$. By evaluating degrees, we see that it is possible that

$$
\beta_{n}=\Phi_{0,0}\left(\gamma_{1}\left(\alpha_{n-1}\right)\right)
$$

On the other hand,

$$
\beta_{n} \gamma_{1}\left(\alpha_{n-1}\right) \neq \Phi_{i j}\left(\gamma_{1}\left(\alpha_{n-1}\right)\right) \text {. }
$$

So, to apply the tertiary cohomology argument, we must show

$$
\Phi_{r-1, r-1}\left(\gamma_{1}\left(\alpha_{n-1}\right)\right) \neq \gamma_{2}\left(\alpha_{n-1}\right)
$$

and

$$
\Phi_{0,0}\left(\gamma_{1}\left(\alpha_{n-1}\right)\right) \neq \beta_{n}
$$

However, if it is the case that $\Phi_{r-1, r-1}\left(\gamma_{1}\left(\alpha_{n-1}\right)\right)=\gamma_{2}\left(\alpha_{n-1}\right)$, then we can show $S^{n}\left\{2^{r}\right\}$ is minimal atomic without resorting to tertiary cohomology operations arguments. We simply apply the Section 2.3 argument using $\Phi_{r-1, r-1}$. Hence, if 
$\Phi_{r-1, r-1}\left(\gamma_{1}\left(\alpha_{n-1}\right)\right)=\gamma_{2}\left(\alpha_{n-1}\right)$ we have shown that $S^{n}\left\{2^{r}\right\}$ is minimal atomic. Otherwise, if $\Phi_{r-1, r-1}\left(\gamma_{1}\left(\alpha_{n-1}\right)\right) \neq \gamma_{2}\left(\alpha_{n-1}\right)$, it must be the case that $\Phi_{r-1, r-1}\left(\gamma_{1}\left(\alpha_{n-1}\right)\right)=0$. In this scenario, we are able to show that $S^{n}\left\{2^{r}\right\}$ is minimal atomic only if $\Phi_{0,0}\left(\gamma_{1}\left(\alpha_{n-1}\right)\right) \neq \beta_{n}$. Then $\Phi_{0,0}\left(\gamma_{1}\left(\alpha_{n-1}\right)\right)=0$, and we will be able to use our previous tertiary operation argument.

\section{A filtration of $\Omega^{2} S^{n}$}

The James construction provides a filtration of $\Omega S^{n}$. We shall use this to build a filtration of $\Omega^{2} S^{n}$ with nice properties. Later, we will use this filtration to study the minimal atomicity of $\Omega^{2} S^{n}$ when $n$ is even.

\subsection{The cohomology of $F_{k}$}

Recall that the James construction, $J\left(S^{n-1}\right)$, is the free monoid on $S^{n-1}$ with basepoint the identity. Then, the $k$ th-filtration, $J_{k}\left(S^{n-1}\right)$ is the subspace of words of length at most $k$. Abbreviating $J_{k}\left(S^{n-1}\right)$ by $F_{k}$, we have colim $F_{k} \simeq J\left(S^{n-1}\right)$. We recall since $S^{n-1}$ is a connected CW-complex that $J\left(S^{n-1}\right) \simeq \Omega \Sigma S^{n-1}$. Furthermore, $F_{k} / F_{k-1} \simeq S^{k(n-1)}$. Now, the cofibration $F_{k} \rightarrow F_{k+1}$ induces a long exact sequence in homology,

$$
\cdots \rightarrow \tilde{H}_{*}\left(F_{k-1}\right) \rightarrow \tilde{H}_{*}\left(F_{k}\right) \rightarrow \tilde{H}_{*}\left(S^{k(n-1)}\right) \rightarrow \cdots
$$

Using the fact that $F_{1} \simeq S^{n-1}$, we may proceed inductively to show

$$
\tilde{H}_{*}\left(F_{k}\right)= \begin{cases}\mathbb{Z} / 2 \mathbb{Z}, & \text { if } *=i(n-1) \text { for } 1 \leqslant i \leqslant k ; \\ 0, & \text { otherwise }\end{cases}
$$

and

$$
\tilde{H}_{*}\left(F_{k-1}\right) \rightarrow \tilde{H}_{*}\left(F_{k}\right) \text { is a monomorphism. }
$$

In this case, $\tilde{H}_{*}\left(F_{k-1}\right) \rightarrow \tilde{H}_{*}\left(\Omega S^{n}\right)$ is a monomorphism of coalgebras, and thus $H^{*}\left(\Omega S^{n}\right) \rightarrow H^{*}\left(F_{k}\right)$ is an epimorphism of algebras.

By taking the vector space dual of the computation of $\tilde{H}_{*}\left(F_{k}\right)$ above, we see there is one generator of $H^{*}\left(F_{2^{k}-1}\right)$ in dimensions $i(n-1)$ for $0 \leqslant i \leqslant 2^{k}-1$. These generators can be identified with $\gamma_{i}\left(\alpha_{n-1}\right) \in H^{i(n-1)}\left(\Omega S^{n}\right)$ for $0 \leqslant i \leqslant 2^{k}-1$. Thus,

$$
H^{*}\left(F_{2^{k}-1}\right)=E\left[\gamma_{1}\left(\alpha_{n-1}\right), \gamma_{2}\left(\alpha_{n-1}\right), \ldots, \gamma_{2^{k-1}}\left(\alpha_{n-1}\right)\right] .
$$

We observe that $H^{*}\left(F_{2^{k}-1}\right)$ inherits the Steenrod algebra structure of $H^{*}\left(\Omega S^{n}\right)$ via the map $H^{*}\left(\Omega S^{n}\right) \rightarrow H^{*}\left(F_{2^{k}-1}\right)$. Thus, the Steenrod operations act trivially on $H^{*}\left(F_{2^{k}-1}\right)$.

\subsection{The cohomology of $Y_{k}$}

Let $Y_{k}$ be the homotopy fiber of $F_{2^{k}-1} \rightarrow F_{2^{k+1}-1}$.

Proposition 6.1. $H^{*}\left(Y_{k}\right)=\Gamma\left[\sigma \gamma_{2^{k}\left(\alpha_{n-1}\right)}\right]$. 
Homology, Homotopy and Applications, vol. 9(1), 2007

We prove Proposition 6.1 using the Eilenberg-Moore spectral sequence.

$$
\begin{aligned}
E_{2} & =\operatorname{Tor}_{H^{*}\left(F_{2^{k+1}-1}\right)}\left(H^{*}\left(F_{2^{k}-1}\right), \mathbb{Z} / 2 \mathbb{Z}\right) \\
& =\operatorname{Tor}_{E\left[\gamma_{1}\left(\alpha_{n-1}\right), \ldots, \gamma_{2^{k}}\left(\alpha_{n-1}\right)\right]}\left(E\left[\gamma_{1}\left(\alpha_{n-1}\right), \ldots, \gamma_{2^{k-1}}\left(\alpha_{n-1}\right)\right], \mathbb{Z} / 2 \mathbb{Z}\right)
\end{aligned}
$$

By the Change of Rings Theorem from $[\mathbf{M c}]$, we have

$$
\operatorname{Tor}_{R \otimes S}(R, \mathbb{Z} / 2 \mathbb{Z})=\operatorname{Tor}_{S}(\mathbb{Z} / 2 \mathbb{Z}, \mathbb{Z} / 2 \mathbb{Z}) .
$$

Applied here, we have

$$
\operatorname{Tor}_{E\left[\gamma_{1}\left(\alpha_{n-1}\right), \ldots, \gamma_{2^{k-1}}\left(\alpha_{n-1}\right)\right] \otimes E\left[\gamma_{2^{k}}\left(\alpha_{n-1}\right)\right]}\left(E\left[\gamma_{1}\left(\alpha_{n-1}\right), \ldots, \gamma_{2^{k-1}}\left(\alpha_{n-1}\right)\right], \mathbb{Z} / 2 \mathbb{Z}\right)
$$

evaluates to

$$
\operatorname{Tor}_{E\left[\gamma_{2^{k}}\left(\alpha_{n-1}\right)\right]}(\mathbb{Z} / 2 \mathbb{Z}, \mathbb{Z} / 2 \mathbb{Z})=\Gamma\left[\sigma \gamma_{2^{k}}\left(\alpha_{n-1}\right)\right] .
$$

Inspection shows that there are no dimensional candidates for non-trivial differentials on these generators, so $E_{2}=E \infty$.

We may conclude that modulo extensions, $H^{*}\left(Y_{k}\right)=\Gamma\left[\sigma \gamma_{2^{k}}\left(\alpha_{n-1}\right)\right]$. Let us denote $\sigma \gamma_{2^{k}}\left(\alpha_{n-1}\right)$ by $\zeta_{k}$. To show that as an algebra $H^{*}\left(Y_{k}\right)=\Gamma\left[\zeta_{k}\right]$, we examine the Serre cohomology spectral sequence applied to $Y_{k} \rightarrow F_{2^{k}-1} \rightarrow F_{2^{k+1}-1}$. Dimensionally, for each $\gamma_{i}\left(\zeta_{k}\right)$ there must be a differential $d_{r}$ such that

$$
d_{r}\left(\gamma_{i}\left(\zeta_{k}\right)\right)=\gamma_{i-1}\left(\zeta_{k}\right) \otimes \gamma_{2^{k}}\left(\alpha_{n-1}\right) .
$$

An inductive proof on $m$ shows that

$$
\gamma_{i}\left(\zeta_{k}\right) \cdot \gamma_{j}\left(\zeta_{k}\right)=\left(\begin{array}{c}
i+j \\
i
\end{array}\right) \gamma_{i+j}\left(\zeta_{k}\right)
$$

where $i+j=m$. We assume that for all $i+j=m-1$,

$$
\gamma_{i}\left(\zeta_{k}\right) \cdot \gamma_{j}\left(\zeta_{k}\right)=\left(\begin{array}{c}
i+j \\
i
\end{array}\right) \gamma_{i+j}\left(\zeta_{k}\right) .
$$

Given $i, j$ such that $i+j=m$, using the Leibniz rule, we have

$$
\begin{aligned}
d_{r}\left(\gamma_{i}\left(\zeta_{k}\right) \cdot \gamma_{j}\left(\zeta_{k}\right)\right)= & \gamma_{i-1}\left(\zeta_{k}\right) \cdot \gamma_{j}\left(\zeta_{k}\right) \otimes \gamma_{2^{k}}\left(\alpha_{n-1}\right)+ \\
& \gamma_{i}\left(\zeta_{k}\right) \cdot \gamma_{j-1}\left(\zeta_{k}\right) \otimes \gamma_{2^{k}}\left(\alpha_{n-1}\right) \\
= & \left(\begin{array}{c}
m-1 \\
j
\end{array}\right) \gamma_{m-1}\left(\zeta_{k}\right) \otimes \gamma_{2^{k}}\left(\alpha_{n-1}\right)+ \\
& \left(\begin{array}{c}
m-1 \\
j-1
\end{array}\right) \gamma_{m-1}\left(\zeta_{k}\right) \otimes \gamma_{2^{k}}\left(\alpha_{n-1}\right) \\
= & \left(\begin{array}{c}
i+j \\
i
\end{array}\right) \gamma_{i+j-1}\left(\zeta_{k}\right) \otimes \gamma_{2^{k}}\left(\alpha_{n-1}\right) .
\end{aligned}
$$

It must be the case that

$$
\gamma_{i}\left(\zeta_{k}\right) \cdot \gamma_{j}\left(\zeta_{k}\right)=\left(\begin{array}{c}
i+j \\
i
\end{array}\right) \gamma_{i+j}\left(\zeta_{k}\right)
$$

since $d_{r}$ takes the same value on both. Then, $H^{*}\left(Y_{k}\right)$ is a divided polynomial algebra on $\zeta_{k}$. 


\subsection{The cohomology of $G_{k}$}

We denote $G_{k}=\Omega F_{2^{k}-1}$.

Proposition 6.2. $H^{*}\left(G_{k}\right)=\bigotimes_{i=0}^{k-1} \Gamma\left[\lambda_{i}\right]$ where $\left|\lambda_{i}\right|=2^{i}(n-1)-1$.

We show Proposition 6.2 by dualizing $H_{*}\left(G_{k}\right)=P\left[l_{0}, \ldots, l_{k-1}\right]$. This homology computation can be proved by induction by utilizing the following Proposition:

Proposition 6.3. In a spectral sequence of coalgebras, given the smallest $r$ such that $d^{r} \neq 0$, it must be the case that for the smallest degree element $x$ with $d^{r}(x) \neq 0$, $d^{r}(x)$ must be primitive.

Then, by dualizing, we have that as vector spaces $H^{*}\left(G_{k}\right)=\Gamma\left[\left(l_{0}\right)^{*},\left(l_{1}\right)^{*}, \ldots\right.$, $\left.\left(l_{k-1}\right)^{*}\right]$. Consider the cohomology Serre spectral sequence applied to the fibration $G_{k-1} \rightarrow G_{k} \rightarrow Y_{k-1}$. As vector spaces

$$
\begin{aligned}
E_{2} & =H^{*}\left(G_{k-1}\right) \otimes H^{*}\left(Y_{k-1}\right) \\
& =\bigotimes_{i=0}^{k-2} \Gamma\left[\lambda_{i}\right] \otimes \Gamma\left[\zeta_{k-1}\right] .
\end{aligned}
$$

But, the spectral sequence converges to the cohomology of $H^{*}\left(G_{k}\right)$ and $E_{2}$ agrees with the cohomology of $H^{*}\left(G_{k}\right)$ as a vector space. Then, $E_{2}=E_{\infty}$. We may identify $\zeta_{k-1}$ with $\lambda_{k-1}$ under $H_{*}\left(G_{k}\right) \rightarrow H_{*}\left(Y_{k-1}\right)$ and so, we have a splitting of algebras,

$$
H^{*}\left(G_{k}\right)=H^{*}\left(G_{k-1}\right) \otimes H^{*}\left(Y_{k-1}\right),
$$

that is,

$$
H^{*}\left(G_{k}\right)=\bigotimes_{i=0}^{k-1} \Gamma\left[\lambda_{i}\right] .
$$

Now, $\lambda_{k-1}$ is an indecomposable element. So, it is the dual of a primitive element of $H_{*}\left(G_{k}\right)$. The only possibility is that $\left(l_{k-1}\right)^{*}=\lambda_{k-1}$, and so $H_{*}\left(G_{k}\right)$ is primitively generated by the permanent cycles $l_{i}$. We have the desired results. We see from above that $H_{*}\left(G_{k-1)} \hookrightarrow H_{*}\left(G_{k}\right)\right.$. Then,

$$
H_{*}\left(G_{k}\right) \rightarrow H_{*}\left(\Omega^{2} S^{n}\right) \text { is a monomorphism of Hopf algebras }
$$

so that we may identify $l_{i}$ with $r_{i}$. Then,

$$
H^{*}\left(\Omega^{2} S^{n}\right) \rightarrow H^{*}\left(G_{k}\right) \text { is an epimorphism of Hopf algebras. }
$$

We identify $\gamma_{j}\left(\rho_{i}\right)$ with $\gamma_{j}\left(\lambda_{i}\right)$. Thus, $H^{*}\left(G_{k}\right)$ inherits the Steenrod algebra structure of $H^{*}\left(\Omega^{2} S^{n}\right)$ via the map $H^{*}\left(\Omega^{2} S^{n}\right) \rightarrow H^{*}\left(G_{k}\right)$. 


\subsection{The equivalence of $Y_{k}$ with $\Omega S^{2^{k}(n-1)}$}

Note that $H^{*}\left(Y_{k}\right) \simeq H^{*}\left(\Omega S^{2^{k}(n-1)}\right)$. In fact, we will show $Y_{k} \simeq \Omega S^{2^{k}(n-1)}$. Our first aim will be to show that a map between $\Omega S^{2^{k}(n-1)}$ and $Y_{k}$ exists. To accomplish this consider the diagram below where the top horizontal map is the defining fibration for $Y_{k}$, the bottom horizontal map is a standard path fibration, and the middle vertical arrow is the constant map,

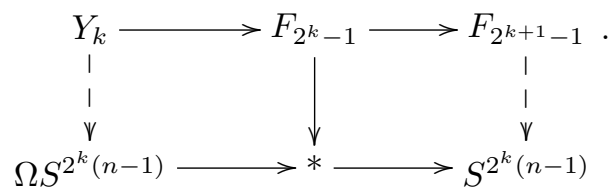

We show that the vertical right arrow exists, thereby giving the existence of a left vertical arrow which makes the whole diagram commute up to homotopy.

Now, it is known that $F_{k}$ is a $\mathrm{CW}$ complex which is a subcomplex of $F_{k+1}$. We recall an explicit description of the cofibration $F_{k} \rightarrow F_{k+1}$. Since $S^{n-1} \simeq D^{n-1} / S^{n-2}$, we have the collapsing map, $D^{n-1} \rightarrow S^{n-1}$. By taking Cartesian products, we have $\left(D^{n-1}\right)^{k+1} \rightarrow\left(S^{n-1}\right)^{k+1}$. Given $x \in\left(D^{n-1}\right)^{k+1}, x$ may be thought of as a word in $D^{n-1}$ with $k+1$ letters, while the boundary of $\left(D^{n-1}\right)^{k+1}$ consists of those words in which at least one letter lies on the boundary of $D^{n-1}$. So, we have a map, $\partial\left(D^{n-1}\right)^{k+1} \rightarrow F_{k}$. But $\left(D^{n-1}\right)^{k+1} \simeq D^{(n-1)(k+1)}$, so we have $S^{(n-1)(k+1)-1} \rightarrow F_{k}$. Then, $F_{k+1}$ is the pushout of the following diagram,

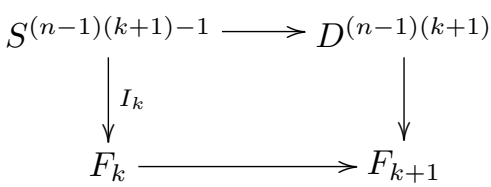

Suppose we have a map $f_{k}: F_{k} \rightarrow X$. A sufficient condition for $f_{k}$ to extend to $f_{k+1}: F_{k+1} \rightarrow X$ is that $f_{k} \circ l_{k}: S^{(n-1)(k+1)-1} \rightarrow X$ is null-homotopic. If so, there exists a homotopy $H: S^{(n-1)(k+1)-1} \times I \rightarrow F_{k}$ from $f_{k} \circ l_{k}$ to the constant basepoint map. Then $H$ induces a map, $D^{(n-1)(k+1)} \rightarrow X$ and by the pushout property, a map $f_{k+1}$ exists with the desired properties. We apply this principle multiple times to obtain the vertical map $F_{2^{k+1}-1} \rightarrow S^{2^{k}(n-1)}$ in (6.2).

Observe that there exists a map $F_{2^{k}} \rightarrow S^{2^{k}(n-1)}$ which maps the word $x_{1} x_{2} \cdots x_{2^{k}}$ to $x_{1} \wedge x_{2} \wedge \cdots \wedge x_{2^{k}}$, where $x_{i} \in S^{n-1}$, and we use $\left(S^{n-1}\right)^{(m)} \simeq S^{m(n-1)}$. Let $f_{2^{k}}$ denote this map, $F_{2^{k}} \rightarrow S^{2^{k}(n-1)}$; the composite $F_{2^{k}-1} \rightarrow F_{2^{k}} \stackrel{f_{2^{k}}}{\longrightarrow} S^{2^{k}(n-1)}$ is trivial (as $F_{2^{k}-1}$ is mapped to the basepoint). We show that extensions $f_{2^{k}+1}, \ldots, f_{2^{k+1}-1}$ exist as in the diagram below,

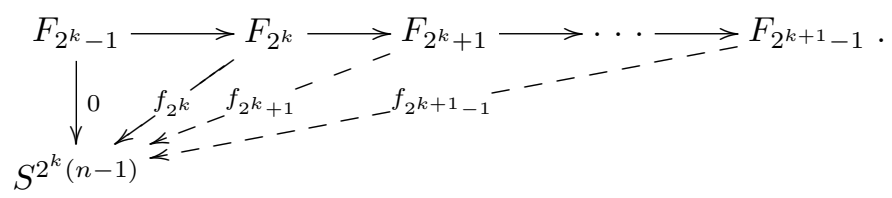

Based on our study of extensions above, for $2^{k}+1 \leqslant i \leqslant 2^{k+1}-1$, to show the 
existence of $f_{i}$, we must examine

$$
f_{i-1} \circ l_{i-1} \in \pi_{(n-1) i-1}\left(S^{2^{k}(n-1)}\right) .
$$

By the Freudenthal suspension theorem[M, p. 83], we know that when

$$
(n-1) i-1<2\left(2^{k}(n-1)\right)-1,
$$

we have an isomorphism

$$
\Sigma: \pi_{(n-1) i-1}\left(S^{2^{k}(n-1)}\right) \rightarrow \pi_{(n-1) i}\left(S^{2^{k}(n-1)+1}\right) .
$$

Since $n>1$,

$$
\begin{aligned}
(n-1) i-1 & \leqslant(n-1) \cdot\left(2^{k+1}-1\right)-1 \\
& =2\left(2^{k}(n-1)\right)-(n-1)-1 \\
& <2\left(2^{k}(n-1)\right)-1 .
\end{aligned}
$$

So we are in the range where $\Sigma: \pi_{(n-1) i-1}\left(S^{2^{k}(n-1)}\right) \rightarrow \pi_{(n-1) i}\left(S^{2^{k}(n-1)+1}\right)$ is an isomorphism. Because we are interested in $f_{k+i} \circ l_{k+i} \in \pi_{(n-1) i-1}\left(S^{2^{k}(n-1)}\right)$, we may investigate the corresponding element in $\pi_{(n-1) i}\left(S^{2^{k}(n-1)+1}\right)$. To do this, we look at what happens to our extension problem when we apply the suspension to our spaces and maps. Consider the diagram,

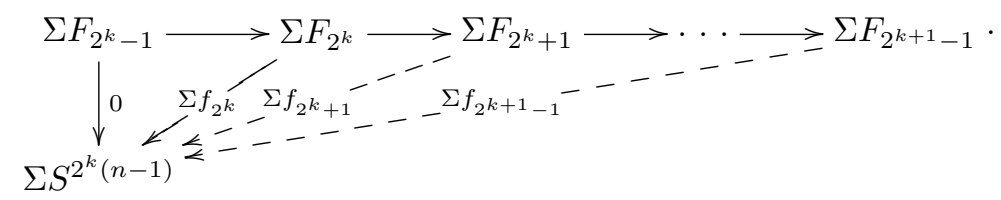

However, for all positive $j$,

$$
\Sigma F_{j} \simeq \bigvee_{i=1}^{j} \sum\left(S^{(n-1)}\right)^{(j)} .
$$

In particular $\Sigma f_{2^{k}-1}: \Sigma F_{2^{k}-1} \rightarrow S^{2^{k}(n-1)+1}$ is the identity on $S^{2^{k}(n-1)+1}$ and maps all other points in the domain to the basepoint. It is clear that the extensions $\Sigma f_{2^{k}+i}$ exist. Similar to $\Sigma f_{2^{k}}, \Sigma f_{2^{k}+i}$ is the identity on $S^{2^{k}(n-1)+1}$ and maps all other points in the domain to the basepoint. So, we have

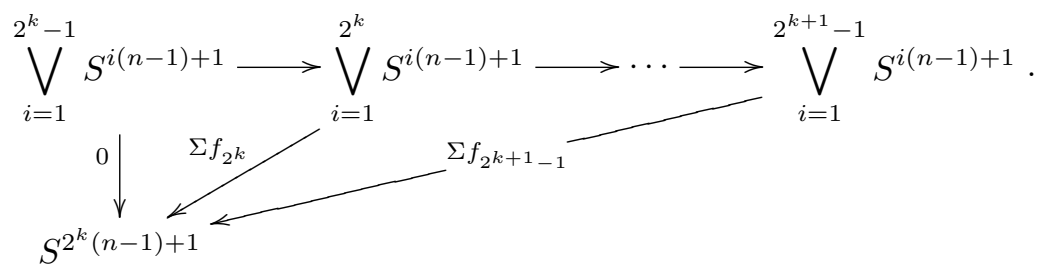

Recall that $l_{k}: S^{(n-1)(k+1)-1} \rightarrow F_{k}$ is the attaching map for $F_{k+1}$ in its CW decomposition. Then, $\Sigma l_{k}$ is the attaching map for $\Sigma F_{k+1}$. We consider the diagram below, where the square is a pushout diagram, 


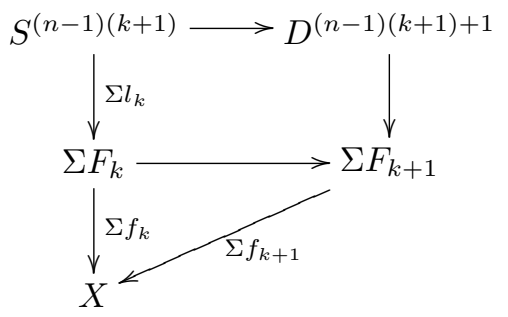

Since the extension $\Sigma f_{k+1}$ exists, we can show that the composite $\Sigma f_{k} \circ \Sigma l_{k}$ must be null-homotopic. We have the composite $\alpha: D^{(n-1)(k+1)} \rightarrow \Sigma F_{k+1} \stackrel{\Sigma f_{k+1}}{\longrightarrow} X$. But, $D^{(n-1)(k+1)-1}$ is just $C S^{(n-1)(k+1)}$. This gives a homotopy from the constant map to the restriction of $\alpha$ to the boundary. This restriction is the map $\Sigma f_{k} \circ \Sigma l_{k}$. Thus, $\Sigma f_{k} \circ \Sigma l_{k} \in \pi_{(n-1)(k+1)}(X)$ corresponds to the zero element.

Applying these ideas to the extensions that exist in (6.4) yields that for $2^{k}+1 \leqslant$ $i \leqslant 2^{k+1}-1$

$$
\Sigma l_{i-1} \circ \Sigma f_{i-1}=0 \in \pi_{(n-1) i}\left(S^{2^{k}(n-1)+1}\right) .
$$

Yet because we are in the range where the Freudenthal suspension is an isomorphism, we have that

$$
l_{i-1} \circ f_{i-1}=0 \in \pi_{(n-1) i-1}\left(S^{2^{k}(n-1)}\right) .
$$

This shows that for $2^{k}+1 \leqslant i \leqslant 2^{k+1}-1, f_{i}$ exists, and hence we have a map

$$
f_{2^{k+1}-1}: F_{2^{k+1}-1} \rightarrow S^{2^{k}(n-1)} \text {. }
$$

Furthermore, by examination of (6.3), we see that the composite

$$
F_{2^{k}-1} \rightarrow F_{2^{k}} \rightarrow \cdots \rightarrow F_{2^{k+1}-1} \rightarrow S^{2^{k}(n-1)}
$$

is null homotopic. So we have shown the existence of a square which commutes up to homotopy, as in the rightmost square of (6.2). Thus, there must exist a map

$$
y_{k}: Y_{k} \rightarrow \Omega S^{2^{k}(n-1)}
$$

such that (6.2) commutes up to homotopy.

Let $E$ denote the cohomology Serre spectral sequence of the fibration

$$
Y_{k} \rightarrow F_{2^{k}} \rightarrow F_{2^{k+1}-1}
$$

Let ' $E$ denote the cohomology Serre spectral sequence of the fibration

$$
\Omega S^{2^{k}(n-1)} \rightarrow * \rightarrow S^{2^{k}(n-1)} .
$$

We have the following commutative diagram.

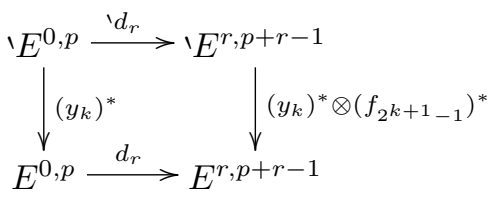


Studying $E$, one can see that there are differentials $d_{r}$ such that

$$
d_{r}\left(\gamma_{i}\left(\zeta_{k}\right)\right)=\gamma_{i-1}\left(\zeta_{k}\right) \otimes \gamma_{2^{k}}\left(\alpha_{n-1}\right) .
$$

As algebras, $H^{*}\left(\Omega S^{2^{k}(n-1)}\right)=\Gamma\left(\alpha_{2^{k}(n-1)-1}\right)$ and $H^{*}\left(S^{2^{k}}(n-1)\right)=E\left[\iota_{2^{k}(n-1)}\right]$. Examining ' $E$ we see there are differentials ' $d_{r}$ such that

$$
d_{r}\left(\gamma_{i}\left(\alpha_{2^{k}(n-1)-1}\right)\right)=\gamma_{i-1}\left(\alpha_{2^{k}(n-1)-1}\right) \otimes \iota_{2^{k}(n-1)} .
$$

Using inductive reasoning, knowing that $\left(f_{2^{k+1}-1}\right)^{*}\left(\gamma_{2^{k}}\left(\alpha_{n-1}\right)\right)=\iota_{2^{k}(n-1)}$, yields the following diagram of elements.

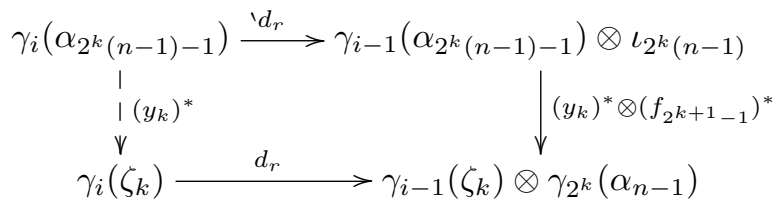

As depicted, it must be the case that

$$
\left(y_{k}\right)^{*}\left(\gamma_{i}\left(\alpha_{2^{k}(n-1)-1}\right)\right)=\gamma_{i}\left(\zeta_{k}\right)
$$

It follows that $\left(y_{k}\right)^{*}$ is an isomorphism in cohomology. Thus, since our spaces are localized at the prime 2 , we have that $y_{k}$ is an equivalence and

$$
Y_{k} \simeq \Omega S^{2^{k}(n-1)}
$$

In particular, the splitting of algebras in (6.1) (with $k$ replaced by $k+1$ ) becomes

$$
H^{*}\left(G_{k+1}\right)=H^{*}\left(G_{k}\right) \otimes H^{*}\left(\Omega S^{2^{k}(n-1)}\right)
$$

with $H^{*}\left(\Omega S^{2^{k}(n-1)}\right)=\Gamma\left[\zeta_{k}\right]$.

\section{Showing $\Omega^{2} S^{n}$ is minimal atomic}

We prove the minimal atomicity of $\Omega^{2} S^{n}$ by breaking the argument into the cases when $n$ is odd and when $n$ is even. The case where $n$ is odd resembles the proof that $\Omega S^{n}$ is minimal atomic. Yet, for $n$ even, there are too many elements to contend with to use previous arguments alone, and we instead combine the methods of higher order cohomology operations with the filtration of Section 6 to obtain the desired conclusion. Finally, we look at the cases when $\Omega^{2} S^{n}$ is not minimal atomic and isolate the elements which are obstructions to minimal atomicity.

\subsection{Spherical candidates of $\Omega^{2} S^{n}$}

From $[\mathbf{K A}]$, we see $H_{*}\left(\Omega^{2}\left(S^{n}\right)\right)$ is a polynomial ring having generators $r_{0}$ and $Q^{J}\left(r_{0}\right)$ where $\left|r_{0}\right|=n-2$ and $Q^{J}$ are the Dyer-Lashof operations, where $J$ is an admissible sequence such that $e(J)>n-2$ and $l(J)<n$. By an induction argument, we can show that any admissible $J$ 's satisfying these constraints must be of the form 
Homology, Homotopy and Applications, vol. 9(1), 2007

$$
\left((n-1) 2^{k},(n-1) 2^{k-1},(n-1) 2^{k-2}, \ldots,(n-1)\right) .
$$

We refer to the generator $Q^{J}\left(r_{0}\right)$ with

$$
J=\left((n-1) 2^{k},(n-1) 2^{k-1},(n-1) 2^{k-2}, \ldots,(n-1)\right)
$$

by $r_{k+1}$. Using the Nishida relations, we can compute the action of the dual of the Steenrod algebra on $H_{*}\left(\Omega^{2} S^{n}\right)$ to obtain the result as in [C, p. 29]:

$$
S q_{*}^{2^{s}}\left(\left(r_{k}\right)^{2^{t}}\right)= \begin{cases}0 & \text { if } s \neq t \text { or } k=0 \text { or if } n \text { even with } s=t \text { and } k=1 \\ r_{k-1}^{2^{s+1}} & \text { if } s=t \text { and } k>1 \text { for } n \text { even or if } s=t \text { and } k \geqslant 1 \\ & \text { for } n \text { odd }\end{cases}
$$

Now, $H^{*}\left(\Omega^{2}\left(S^{n}\right)\right)=\bigotimes_{i \geqslant 0, j \geqslant 0} P\left[\gamma_{2^{i}}\left(\rho_{j}\right)\right] /\left(\gamma_{2^{i}}\left(\rho_{j}\right)\right)^{2}$ where the dual of $\left(r_{j}\right)^{2^{i}}$ is $\gamma_{2^{i}}\left(\rho_{j}\right)$. Using this information, we can compute the action of the Steenrod operations on $H^{*}\left(\Omega^{2} S^{n}\right)$ :

$S q^{2^{s}}\left(\gamma_{2^{t}}\left(\rho_{k}\right)\right)= \begin{cases}0 & \text { if } s+1 \neq t, \text { or if } n \text { is even with } s+1=t \text { and } k=0 \\ \gamma_{2^{s}}\left(\rho_{k+1}\right) & \text { if } s+1=t \text { with } n \text { odd, or } s+1=t \text { with } n \text { even and } \\ & k \geqslant 1\end{cases}$

\section{2. $n$ odd}

Now, in the case where $n$ is odd, each $\left(r_{k}\right)^{2^{t}}$ is not an $A$-annihilated primitive when $k \geqslant 1$. Thus, these elements cannot be spherical. The only candidates for spherical elements above the Hurewicz dimension are $\left(r_{0}\right)^{2^{t}}$ for $t \geqslant 1$. We shall refer to these elements as $p_{t}$. We apply the techniques of higher order cohomology operations to prove that $p_{t}$ is not spherical. For each $t>0$, we exhibit a higher order cohomology operation whose image includes $\gamma_{2^{t}}\left(\rho_{0}\right)$.

Let us establish how the Steenrod operations act on $\gamma_{2^{t}}\left(\rho_{0}\right)$ for $t \geqslant 0$. First, for $t=0$, we see from above that no Steenrod operation of the form $S q^{2^{i}}$ acts nontrivally on $\gamma_{1}\left(\rho_{0}\right)$. Since operations of the type $S q^{2^{i}}$ generate the Steenrod algebra, we conclude that all Steenrod operations annihilate $\gamma_{1}\left(\rho_{0}\right)$.

For $t>0$, the only generator of the Steenrod algebra which acts non-trivially on $\gamma_{2^{t}}\left(\rho_{0}\right)$ is $S q^{2^{t-1}}$; the result of this action is $\gamma_{2^{t-1}}\left(\rho_{1}\right)$. If $t-1>0$, again, we have exactly one generator of the Steenrod algebra which acts non-trivially on $\gamma_{2^{t-1}}\left(\rho_{1}\right)$, $S q^{2^{t-2}}$. Proceeding in this fashion, we see that the Steenrod operations which act non-trivially on $\gamma_{2^{t}}\left(\rho_{0}\right)$ are of the form $S q^{I}$ where $I=\left(2^{m}, 2^{m+1}, \ldots, 2^{t-1}\right)$ where $0 \leqslant m \leqslant t-1$. In particular, the highest dimensional Steenrod operation which can act on $\gamma_{2^{t}}\left(\rho_{0}\right)$ is of degree $1+2+\ldots+2^{t-1}=2^{t}-1$.

\subsubsection{Showing $p_{t+1}$ is not spherical when $t>0$}

Consider the relation $S q^{2^{t}(n-2)+1}=S q^{1} S q^{2^{t}(n-2)}$ which is similar to (4.2) with $n-1$ replaced by $n-2$ when $t>0$. This relation gives rise to a secondary cohomology operation which we shall call $\Theta_{t}$. Then, for appropriate $\theta$ and $\gamma$,

$$
\Theta_{t}(\tau+\gamma)=\Theta_{t}(\tau)+\Theta_{t}(\gamma)+\tau \gamma
$$


The defining cohomology class $\phi_{2} \in H^{2^{t+1}(n-2)}\left(\Omega E_{2}\right)$ is primitive. $\Theta_{t}$ is defined on elements $\tau \in H^{2^{t}(n-2)}(X)$ for which $S q^{2^{t}(n-2)}$ has a trivial action, and the image

$$
\Theta_{t}(\tau) \in H^{2^{t+1}(n-2)}(X) / S q^{1}\left(H^{2^{t+1}(n-2)-1}(X) .\right.
$$

In particular, we see that $S q^{2^{t}(n-2)}$ acts trivially on $\gamma_{2^{t}}\left(\rho_{0}\right)$ since

$$
\left|\gamma_{2^{t}}\left(\rho_{0}\right)\right|=2^{t}(n-2)
$$

and

$$
\left(\gamma_{2^{t}}\left(\rho_{0}\right)\right)^{2}=0
$$

So, $\Theta_{t}$ is defined on $\gamma_{2^{t}}\left(\rho_{0}\right)$ for $t>0$. Then,

$$
\gamma_{2^{t+1}}\left(\rho_{0}\right) \notin S q^{1}\left(H^{2^{t+1}(n-2)-1}\left(\Omega^{2} S^{n}\right)\right) .
$$

For $t>0, \gamma_{2^{t+1}}\left(\rho_{0}\right)$ clearly is not in the image of $S q^{1}$ and does not belong to the indeterminacy of $\Theta_{t}$.

Let $f: \Omega S^{n-1} \rightarrow \Omega^{2} S^{n}$ be the result of looping the canonical map $\eta$. Then, $f$ maps the indecomposable elements $\gamma_{2^{t}}\left(\rho_{0}\right)$ of $\Omega^{2} S^{n}$ to the indecomposable elements $\gamma_{2^{t}}\left(\alpha_{n-2}\right)$ of $\Omega S^{n-1}$. The following diagram commutes:

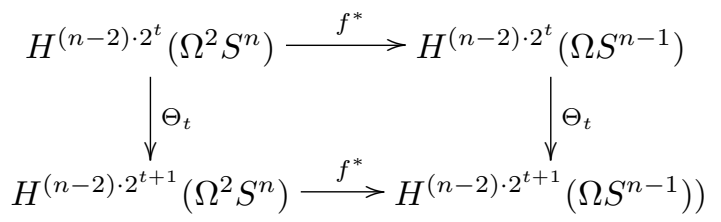

Now, $\Theta_{t}\left(f^{*}\left(\gamma_{2^{t}}\left(\rho_{0}\right)\right)=\gamma_{2^{t+1}}\left(\alpha_{n-2}\right)\right.$. By chasing the diagram, we see that modulo $S q^{1}\left(H^{2^{t+1}(n-2)-1}\left(\Omega^{2} S^{n}\right)\right)$, we have $\Theta_{t}\left(\gamma_{2^{t}}\left(\rho_{0}\right)\right)=\gamma_{2^{t+1}}\left(\rho_{0}\right)+\tau$ where $\tau$ is in the kernel of $H^{(n-2) 2^{t+1}}\left(\Omega^{2} S^{n}\right) \rightarrow H^{(n-2) 2^{t+1}}\left(\Omega S^{n-1}\right) ; \tau$ must be a decomposable element since the only elements $\tau$ of degree $(n-2) 2^{t+1}$ besides $\gamma_{2^{t+1}}\left(\rho_{0}\right)$ are decomposable elements of $H^{(n-2) 2^{t+1}}\left(\Omega^{2} S^{n}\right)$. So $\tau=\sum a_{i} b_{i}$ where $\left|a_{i}\right|,\left|b_{i}\right|>0$. As we have noted the module of indeterminacy $S q^{1}\left(H^{(n-1) 2^{t+1}-1}\left(\Omega^{2} S^{n}\right)\right)$ does not contain $\gamma_{2^{t+1}}\left(\rho_{0}\right)$, so it must contain only decomposables. Thus,

$$
\Theta_{t}\left(\gamma_{2^{t}}\left(\rho_{0}\right)\right)=\gamma_{2^{t+1}}\left(\rho_{0}\right)+\tau
$$

where $\tau \in H^{(n-2) 2^{t+1}}\left(\Omega^{2} S^{n}\right)$ is a sum of decomposable elements (possibly zero).

Appealing to Section 2.3, we have for $t>0, p_{t+1}$ is not spherical.

7.2.2. Showing $p_{1}$ is not spherical when $n-1 \neq 2^{r}$

We show that $p_{1}$ is not spherical. To accomplish this, we exhibit a higher order cohomology operation which we evaluate on $\gamma_{1}\left(\rho_{0}\right)$ to yield $\gamma_{2}\left(\rho_{0}\right)$. First, we take the case that $n-1$ is not a power of 2. Then, $S q^{n-1}$ has a factorization as in (3.1), with 
Homology, Homotopy and Applications, vol. 9(1), 2007

$$
S q^{n-1}=S q^{a} S q^{2^{r}}+\sum_{c>0}\left(\begin{array}{c}
2^{r}-c-1 \\
a-2 c
\end{array}\right) S q^{(n-1)-c} S q^{c}
$$

where $2^{r}$ is the largest power of 2 which appears in the binomial expansion of $n-1$ and $(n-1)=a+2^{r}$. Given this primary relation, we may consider the Brown and Peterson secondary cohomology operation which stems from this, which we shall call $\Theta_{0} . \Theta_{0}$ is defined on elements of $H^{n-2}(X)$ which are annihilated by the $S q^{c}$ above, and its image lies in $H^{2(n-2)}(X)$ with indeterminacy $\sum S q^{(n-1)-c} H^{n-3+c}(X)$ where the sum runs over those $c$ such that $0 \leqslant c \leqslant\left[\frac{n-1}{2}\right]$ with $\left(\begin{array}{c}2^{r}-c-1 \\ a-2 c\end{array}\right) \neq 0$.

If we set $X=\Omega^{2} S^{n}$, we see $\Theta_{0}$ is defined on $\gamma_{1}\left(\rho_{0}\right)$ because $\gamma_{1}\left(\rho_{0}\right)$ is annihilated by all Steenrod operations. By considering the map, as above, $\Omega S^{n-1} \rightarrow \Omega^{2} S^{n}$, we may show that $\Theta_{0}\left(\gamma_{1}\left(\rho_{0}\right)\right)=\gamma_{2}\left(\rho_{0}\right)+\sum a_{i} b_{i}$ for some $a_{i}, b_{i} \in H^{*}\left(\Omega^{2} S^{n}\right)$ such that $a_{i} b_{i} \in H^{2(n-2)}\left(\Omega^{2} S^{n}\right)$, and $\left|a_{i}\right|,\left|b_{i}\right|>0$. Thus, if we suppose $p_{1}$ is spherical, we must get a contradiction as in Section 2.3.

7.2.3. Showing $p_{1}$ is not spherical when $n-1=2^{r+1}$ for $r \geqslant 3$

Let us take the case when $n-1$ is a power of two greater than 8 . Then, $n-1=2^{r+1}$ for $r \geqslant 3$. As before, we provide a higher cohomology operation which acts on $\gamma_{1}\left(\rho_{0}\right)$ to yield $\gamma_{2}\left(\rho_{0}\right)$. We may apply our tertiary operation $\Psi$ to this situation. First, we check that $\Psi$ is defined on $\gamma_{1}\left(\rho_{0}\right)$ by showing that

1. $S q^{2^{i}}$ annihilates $\gamma_{1}\left(\rho_{0}\right)$ for $i \leqslant r$

2. $\Phi_{i j}$ annihilates $\gamma_{1}\left(\rho_{0}\right)$ for $i, j \leqslant r$

We have already seen that (1) is true. This result enables us to define $\Phi_{i j}\left(\gamma_{1}\left(\rho_{0}\right)\right)$; now we try to compute it. We review the construction of Adams' $\Phi_{i j}$. Recall $\Phi_{i j}$ is a secondary cohomology operation based on a primary relation of the form $\sum_{0 \leqslant m \leqslant j} b_{m} S q^{2^{m}}=0$ where $b_{m}$ is an element of the Steenrod algebra. To obtain this relation, we apply the Adem relation to $S q^{2^{i}} S q^{2^{j}}$ and express the right-hand Steenrod operation of each summand in terms of the Steenrod operations, $S q^{2^{m}}$. We note that each term of this summand has degree $2^{i}+2^{j}$ and $b_{j}=S q^{2^{i}}$.

For any $l$, let $S q^{2^{m}}: K(l) \rightarrow K\left(l+2^{m}\right)$ represent the element $S q^{2^{m}}\left(\kappa_{l}\right)$ where $\kappa_{l}$ is the fundamental class of $K(l)$. Then, consider $f_{1}: K(l) \rightarrow \prod_{0 \leqslant m \leqslant j} K\left(l+2^{m}\right)$ which is given by $f_{1}^{*}\left(\kappa_{l+2^{m}}\right)=S q^{2^{m}}\left(\kappa_{l}\right)$. Denote the homotopy fiber of $f_{1}$ by $A_{1}$. We have the map $g_{1}: A_{1} \rightarrow K(l)$ with fiber $\prod_{0 \leqslant m \leqslant j} K\left(l+2^{m}-1\right)$. The elements $\kappa_{l+2^{m}-1}$ transgress to $S q^{2^{m}}\left(\kappa_{l}\right)$. Then,

$$
\sum_{0 \leqslant m \leqslant j} b_{m} \kappa_{l+2^{m}-1}
$$

transgresses to

$$
\sum_{0 \leqslant m \leqslant j} b_{m} S q^{2^{m}} \kappa_{l}=0
$$

So, $\sum_{0 \leqslant m \leqslant j} b_{m} \kappa_{l+2^{m}-1}$ survives in the Serre spectral sequence of the fibration 
Homology, Homotopy and Applications, vol. 9(1), 2007

$$
\prod_{0 \leqslant m \leqslant j} K\left(l+2^{m}-1\right) \rightarrow A_{1} \stackrel{g_{1}}{\rightarrow} K(l),
$$

and there exists $\phi_{i j} \in H^{2^{i}+2^{j}+l-1}\left(A_{1}\right)$. We may represent this cohomology class by a map $\phi_{i j}: A_{1} \rightarrow K_{2^{i}+2^{j}+l-1}$. If we replace $l$ by $n-1$, effectively, the diagram below captures the action of $\Phi_{i j}$ on cohomology elements in degree $n-1$.

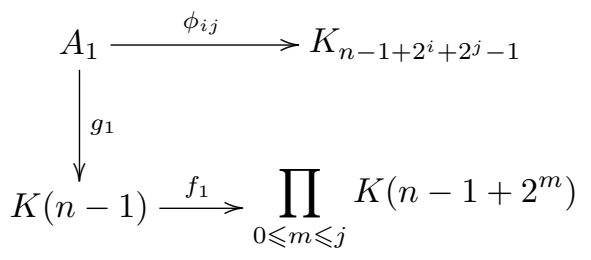

Observe that if we loop this diagram, we will obtain a diagram which represents the action of $\Phi_{i j}$ on cohomology elements in degree $n-2$ because $\Phi_{i j}$ is stable. We have discussed that $\Phi_{i j}$ is defined on $\gamma_{1}\left(\alpha_{n-1}\right) \in H^{n-1}\left(\Omega S^{n}\right)$. By replacing $l$ above by $n-1$ and by representing $\gamma_{1}\left(\alpha_{n-1}\right)$ by the map $f: \Omega S^{n} \rightarrow K(n-1)$, we have that $\Phi_{i j}\left(\gamma_{1} \rho_{1}\right)$ corresponds to $\phi_{i j} \circ \tilde{f}$ :

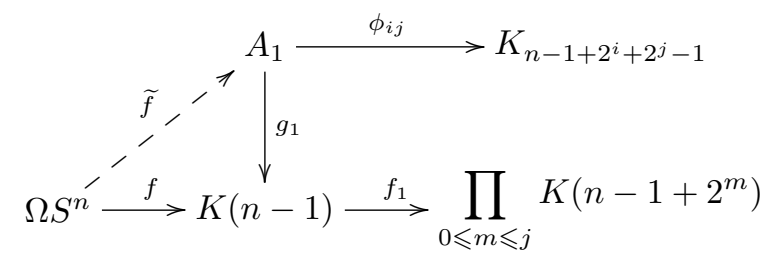

We exhibited earlier that $\Phi_{i j}\left(\gamma_{1}\left(\alpha_{n-1}\right)\right)=0$ with zero indeterminacy. Thus, $\phi_{i j} \circ \tilde{f}$ is null homotopic.

Now, looping this diagram enables us to study

$$
\Phi_{i j}\left(\gamma_{1}\left(\rho_{0}\right)\right)=\Omega \phi_{i j} \circ \Omega \tilde{f}
$$

So

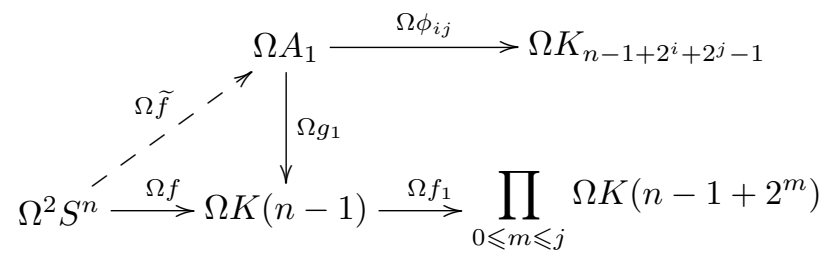

Since $\phi_{i j} \circ \tilde{f}$ is null homotopic, $\Omega \phi_{i j} \circ \Omega \tilde{f}$ is null homotopic. Thus, $\Phi_{i j}\left(\gamma_{1}\left(\rho_{0}\right)\right)$ is zero modulo indeterminacy. Via dimensional arguments, we show the indeterminacy is zero. Now

$$
\Phi_{i j}\left(\gamma_{1}\left(\rho_{0}\right)\right) \in H^{2^{i}+2^{j}-1+n-2}\left(\Omega^{2} S^{n}\right) .
$$

By definition, the indeterminacy of the secondary cohomology operation $\Phi_{i j}$ is 
Homology, Homotopy and Applications, vol.9(1), 2007

$$
\sum_{0 \leqslant m \leqslant r} b_{m} H^{2^{i}+2^{j}-1+n-2-\left|b_{m}\right|}\left(\Omega^{2} S^{n}\right) .
$$

Yet, $\left|b_{m}\right|=2^{i}+2^{j}-2^{m}$, so the indeterminacy lies in

$$
\sum_{0 \leqslant m \leqslant r} b_{m} H^{n-3+2^{m}}\left(\Omega^{2} S^{n}\right)=\sum_{0 \leqslant m \leqslant r} b_{m} H^{2^{r+1}+2^{m}-2}\left(\Omega^{2} S^{n}\right) .
$$

To understand indeterminacy, we consider $H^{2^{r+1}+2^{m}-2}\left(\Omega^{2} S^{n}\right)$ for $0 \leqslant m \leqslant r$. Now, $\gamma_{1}\left(\rho_{0}\right)$ belongs to $H^{2^{r+1}-1}\left(\Omega^{2} S^{n}\right)$, but all other $\gamma_{a}\left(\rho_{b}\right)$, and thus products of the generators of $H^{*}\left(\Omega^{2} S^{n}\right)$, have degree too large to be in $H^{2^{r+1}+2^{m}-2}\left(\Omega^{2} S^{n}\right)$. Since $\gamma_{1}\left(\rho_{0}\right)$ is annihilated by all Steenrod operations $b_{0}$, the indeterminacy must be zero. Thus, $\Phi_{i j}\left(\gamma_{1}\left(\rho_{0}\right)\right)$ is zero with zero indeterminacy for each $i, j$ with $0 \leqslant i \leqslant j \leqslant r$ and $i \neq j+1$. Since $S q^{2^{i}}$ annihilates $\gamma_{1}\left(\rho_{0}\right)$ for $i \leqslant r$, we may proceed in applying the tertiary operation $\Psi$ to $\gamma_{1}\left(\rho_{0}\right)$.

Again, we use the map $f: \Omega S^{n-1} \rightarrow \Omega^{2} S^{n}$ noting that $f^{*}\left(\gamma_{2^{t}}\left(\rho_{0}\right)\right)=\gamma_{2^{t}}\left(\alpha_{n-2}\right)$. The following diagram commutes:

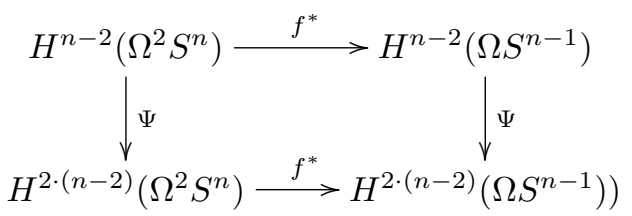

Now, $\Psi\left(f^{*}\left(\gamma_{1}\left(\rho_{0}\right)\right)\right)=\gamma_{2}\left(\alpha_{n-2}\right)$ modulo zero indeterminacy. By chasing the diagram, we see that modulo the indeterminacy of $\Psi, \Psi\left(\gamma_{1}\left(\rho_{0}\right)\right)=\gamma_{2}\left(\rho_{0}\right)+\tau$, where $\tau \in$ kernel of $H^{(n-2) \cdot 2}\left(\Omega^{2} S^{n}\right) \rightarrow H^{(n-2) \cdot 2}\left(\Omega S^{n-1}\right)$. However, there are no elements of degree $(n-2) \cdot 2$ in $H^{*}\left(\Omega^{2} S^{n}\right)$ besides $\gamma_{2}\left(\rho_{0}\right)$. So $\tau$ must be zero. Also, $\gamma_{2}\left(\rho_{0}\right)$ is not in the image of the Steenrod algebra action on $H^{*}\left(\Omega^{2} S^{n}\right)$ so $\gamma_{2}\left(\rho_{0}\right)$ is not in the indeterminacy of $\Psi$. Hence for dimension reasons the indeterminacy module of $\Psi$ must be zero, modulo zero,

$$
\Psi\left(\gamma_{1}\left(\rho_{0}\right)\right)=\gamma_{2}\left(\rho_{0}\right) .
$$

Thus, following the ideas of Section 2.3, $p_{1}$, the dual element of $\gamma_{2}\left(\rho_{0}\right)$, is not spherical. Thus, we have shown that in the case when $n$ is odd and $n-1 \neq 2^{r}$ where $r>3, \Omega^{2} S^{n}$ is minimal atomic.

\section{3. $n$ even}

To determine which elements of $H_{*}\left(\Omega^{2} S^{n}\right)$ are spherical when $n$ is even, we determine which primitive elements are annihilated by the dual of the Steenrod algebra. From our work above, we see that the only elements fitting this description are $\left(r_{0}\right)^{2^{t}}$ and $\left(r_{1}\right)^{2^{t}}$ for $t \geqslant 0$. Thus, we establish how the Steenrod operations act on $\gamma_{2^{t}}\left(\rho_{0}\right)$ and $\gamma_{2^{t}}\left(\rho_{1}\right)$ so we may use our higher order cohomology techniques.

We see from our previous work that $S q^{2^{s}}\left(\gamma_{2^{t}}\left(\rho_{0}\right)\right)=0$ and so the entire Steenrod algebra annihilates $\gamma_{2^{t}}\left(\rho_{0}\right)$. Also, $\gamma_{1}\left(\rho_{1}\right)$ has a trivial action under the Steenrod algebra. 
For $t>0$, the only generator of the Steenrod algebra which acts non-trivially on $\gamma_{2^{t}}\left(\rho_{1}\right)$ is $S q^{2^{t-1}}$ yielding $\gamma_{2^{t-1}}\left(\rho_{2}\right)$. If $t-1>0$, again, we have exactly one generator of the Steenrod algebra which acts non-trivially on $\gamma_{2^{t-1}}\left(\rho_{1}\right), S q^{2^{t-2}}$. Proceeding in this fashion, we see that the Steenrod algebra elements that act non-trivially on $\gamma_{2^{t}}\left(\rho_{1}\right)$ are of the form $S q^{I}$ where $I=\left(2^{m}, 2^{m+1}, \ldots, 2^{t-1}\right)$ where $0 \leqslant m \leqslant t-1$. In particular, the highest dimensional Steenrod operation which can act on $\gamma_{2^{t}}\left(\rho_{1}\right)$ is of degree $1+2+\ldots+2^{t-1}=2^{t}-1$.

As in Section 7.2, we use the same secondary cohomology operation $\Theta_{t}$ defined on elements, $\gamma \in H^{2^{t}(n-2)}(X)$ such that $S q^{2^{t}(n-2)}(\gamma)=0$ with image contained in $H^{2^{t+1}(n-2)}(X) / S q^{1}\left(H^{2^{t+1}}(n-2)-1(X)\right)$. Then, as before, for $t>0, \Theta_{t}$ is defined on $\gamma_{2^{t}}\left(\rho_{0}\right)$ and $\gamma_{2^{t+1}}\left(\rho_{0}\right) \notin S q^{1}\left(H^{2^{t+1}(n-2)-1}\left(\Omega^{2} S^{n}\right)\right)$. That is, for $t>0, \gamma_{2^{t+1}}\left(\rho_{0}\right)$ does not belong to the indeterminacy of $\Theta_{t}$.

7.3.1. Showing $\left(r_{0}\right)^{2^{t}}$ is not spherical for $t>0$

Again, we use $f: \Omega S^{n-1} \rightarrow \Omega^{2} S^{n}$ noting that $f^{*}\left(\gamma_{2^{t}}\left(\rho_{0}\right)\right)=\gamma_{2^{t}}\left(\alpha_{n-2}\right)$. The following diagram commutes:

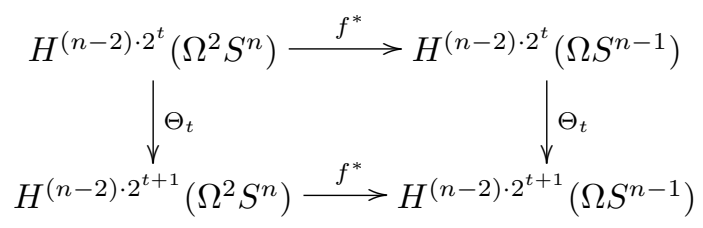

As in the case when $n$ is odd, chasing the diagram leads to the conclusion that $\Theta_{t}\left(\gamma_{2^{t}}\left(\rho_{0}\right)\right)=\gamma_{2^{t+1}}\left(\rho_{0}\right)+\tau$ where $\tau \in H^{(n-2) 2^{t+1}}\left(\Omega^{2} S^{n}\right)$ is a sum of decomposable elements (possibly zero). Thus, using Section 2.3 for $t>0,\left(r_{0}\right)^{2^{t+1}}$ is not spherical.

To show $\left(r_{0}\right)^{2}$ is not spherical, we observe that in the case when $n$ is even, we can factor $S q^{n-1}$. Further, $\gamma_{2}\left(\rho_{0}\right)$ is annihilated by the Steenrod algebra and is not in the image of the Steenrod algebra. Thus, repeating the argument in Section 7.2.2 shows that $\gamma_{2}\left(\rho_{0}\right)+\sum a_{i} b_{i}$ is in the image of $\Theta_{0}$. Thus, $\left(r_{0}\right)^{2}$ is not spherical and for all $t>0,\left(r_{0}\right)^{2^{t}}$ is not spherical.

7.3.2. Showing $\left(r_{1}\right)^{2^{t}}$ is not spherical when $t>0$

Consider the Steenrod algebra relation,

$$
S q^{2^{t}(2(n-1)-1)+1}=S q^{1} S q^{2^{t}(2(n-1)-1)} .
$$

Analogous to the results of Section 4.2, for each $t$ this relation gives rise to a secondary cohomology operation, which we shall denote $\Phi_{t}$. In particular, $\Phi_{t}$ is defined on $H^{*}\left(\Omega S^{2(n-1)}\right)=H^{*}\left(Y_{1}\right)$ with zero indeterminacy and similar to (4.3) we obtain

$$
\Phi_{t}\left(\gamma_{2^{t}}\left(\rho_{1}\right)\right)=\gamma_{2^{t+1}}\left(\rho_{1}\right)
$$

Considering $\gamma_{2^{t}}\left(\rho_{1}\right)$ as an element of $H^{*}\left(\Omega^{2} S^{n}\right)$, we deduced in Section 7.3 that the highest dimensional Steenrod operation which acts non-trivially on $\gamma_{2^{t}}\left(\rho_{1}\right)$ has degree $2^{t}-1$. Then $S q^{2^{t}(2(n-1)-1)}$ must act trivially on $\gamma_{2^{t}}\left(\rho_{1}\right)$. Since $H^{*}\left(G_{2}\right)$ 
inherits its Steenrod algebra action from $H^{*}\left(\Omega^{2} S^{n}\right), S q^{2^{t}(2(n-1)-1)}$ acts trivially on $\gamma_{2^{t}}\left(\rho_{1}\right)$ considered as an element of $H^{*}\left(G_{2}\right)$. Thus $\Phi_{t}\left(\gamma_{2^{t}}\left(\rho_{1}\right)\right)$ is defined on $H^{*}\left(\Omega^{2} S^{n}\right)$ and $H^{*}\left(G_{2}\right)$.

Let $g_{1}: G_{2} \rightarrow Y_{1}$ be the map which occurs in the fibration $G_{1} \rightarrow G_{2} \rightarrow Y_{1}$. In our Serre spectral sequence analysis, we saw that $\left(g_{1}\right)^{*}: H^{*}\left(Y_{1}\right) \hookrightarrow H^{*}\left(G_{2}\right)$ and so $\left(g_{1}\right)^{*}\left(\gamma_{2^{i}}\left(\rho_{1}\right)\right)=\gamma_{2^{i}}\left(\rho_{1}\right)$. Then, by naturality, we have a diagram

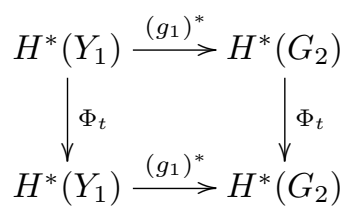

Then, $\Phi_{t}\left(\left(g_{1}\right)^{*}\left(\gamma_{2^{t}}\left(\rho_{1}\right)\right)\right)=\left(g_{1}\right)_{*}\left(\Phi_{t}\left(\gamma_{2^{t}}\left(\rho_{1}\right)\right)\right)$. So, modulo indeterminacy in $H^{*}\left(G_{2}\right)$,

$$
\Phi\left(\gamma_{2^{t}}\left(\rho_{1}\right)\right)=\gamma_{2^{t+1}}\left(\rho_{1}\right) .
$$

Since $H^{*}\left(G_{2}\right)$ inherits its Steenrod algebra structure from $H^{*}\left(\Omega^{2} S^{n}\right)$ we can see that $\gamma_{2^{t+1}}\left(\rho_{1}\right)$ is not in the image of $S q^{1}$. Furthermore, there are no indecomposable elements of $H^{*}\left(\Omega^{2} S^{n}\right)$ with degree $2^{t+1}(2(n-1)-1)=\left|\gamma_{2^{t+1}}\left(\rho_{1}\right)\right|$. So, only decomposables are in the indeterminacy of $\Phi_{t}, S q^{1}\left(H^{2^{t+1}(2(n-1)-1)-1}\left(G_{2}\right)\right)$.

Using the map $g_{2}: G_{2} \rightarrow \Omega^{2} S^{n}$, we have following commutative diagram.

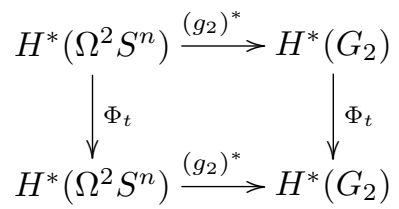

Then, modulo the indeterminacy of $S q^{1}\left(H^{2^{t+1}(2(n-1)-1)-1}\left(\Omega^{2} S^{n}\right)\right)$,

$$
\begin{aligned}
\left(\left(g_{2}\right)^{*}\right)\left(\Phi_{t}\left(\gamma_{2^{t}}\left(\rho_{1}\right)\right)\right) & =\Phi_{t}\left(\left(g_{2}\right)^{*}\left(\gamma_{2^{t}}\left(\rho_{1}\right)\right)\right) \\
& =\Phi_{t}\left(\gamma_{2^{t}}\left(\rho_{1}\right)\right)=\gamma_{2^{t+1}}\left(\rho_{1}\right) .
\end{aligned}
$$

As before, we observe that $\gamma_{2^{t+1}}\left(\rho_{1}\right)$ does not belong to the indeterminacy for $\Phi_{t}$ defined on $H^{*}\left(\Omega^{2} S^{n}\right)$. Also, the only elements that belong to the indeterminacy must be decomposables. The kernel of

$$
\left(g_{2}\right)^{*}: H^{2^{t+1}(2(n-1)-1)}\left(\Omega^{2} S^{n}\right) \rightarrow H^{2^{t+1}(2(n-1)-1)}\left(G_{2}\right)
$$

consists of decomposable elements. Thus, it must be the case that for some decomposable element $\tau \in H^{*}\left(\Omega^{2} S^{n}\right)$

$$
\Phi_{t}\left(\gamma_{2^{t}}\left(\rho_{1}\right)\right)=\gamma_{2^{t+1}}\left(\rho_{1}\right)+\tau
$$

Using Section 2.3, we see that $\left(r_{1}\right)^{2^{t+1}}$ cannot be spherical.

To show that $\left(r_{1}\right)^{2}$ is not spherical, we refer to Section 4.2 replacing $n$ with $\left|r_{1}\right|=2(n-1)$. We observe that since $n$ is even, $2(n-1)$ is not a power of 2 , and 
a factorization of $S q^{2(n-1)}$ exists. Thinking of $\gamma_{1}\left(\rho_{1}\right), \gamma_{2}\left(\rho_{2}\right)$ as elements of $H^{*}\left(Y_{1}\right)$, the argument of Section 4.2 shows that

$$
\Phi_{0}\left(\gamma_{1}\left(\rho_{1}\right)\right)=\gamma_{2}\left(\rho_{1}\right)+\sum a_{i} b_{i} .
$$

We would like a version of (7.3) to hold in $H^{*}\left(G_{2}\right)$ and $H^{*}\left(\Omega^{2} S^{n}\right)$. Observe that $\gamma_{1}\left(\rho_{1}\right) \in H^{*}\left(G_{2}\right)$ is annihilated by all the Steenrod operations, so $\Phi_{0}$ can legitimately be defined on $\gamma_{1}\left(\rho_{1}\right)$. Since $\gamma_{2}\left(\rho_{1}\right) \in H^{*}\left(G_{2}\right)$ is not in the image of any Steenrod operations, $\gamma_{2}\left(\rho_{1}\right)$ will not be cancelled out when taking into account indeterminacy of $\Phi_{0}$ defined on $H^{*}\left(G_{2}\right)$. Then using naturality of $\Phi_{0}$ applied to $g_{1}$, we have that modulo the indeterminacy of $H^{*}\left(G_{2}\right),(7.3)$ holds. Applying naturality of $\Phi_{0}$ with respect to $g_{2}$, we obtain (7.3) thinking of $\gamma_{1}\left(\rho_{1}\right), \gamma_{2}\left(\rho_{1}\right)$ as elements of $H^{*}\left(\Omega^{2} S^{n}\right)$. Thus, it must be the case that $\left(r_{1}\right)^{2}$ is not spherical, and thus for all $t>0,\left(r_{1}\right)^{2^{t}}$ is not spherical.

7.3.3. Showing $r_{1}$ is not spherical when $n$ is not a power of 2

We show that $\gamma_{1}\left(\rho_{0}\right)$ hits $\gamma_{1}\left(\rho_{1}\right)$ via a higher order cohomology operation. We suppose that $n$ is not a power of 2 . For $k \geqslant 2$, let us take the adjoint of the identity map on $\Omega F_{2^{k}-1}, f: \Sigma G_{k} \rightarrow F_{2^{k}-1}$. Then,

$$
f^{*}\left(\gamma_{2^{i}}\left(\alpha_{n-1}\right)\right)=\Sigma \gamma_{1}\left(\sigma \gamma_{2^{i}}\left(\alpha_{n-1}\right)\right)=\Sigma \gamma_{1}\left(\rho_{i}\right) .
$$

We have shown that there exist secondary cohomology operations $\Phi_{i}$ such that $\Phi_{0}\left(\gamma_{1}\left(\alpha_{n-1}\right)\right)=\gamma_{2}\left(\alpha_{n-1}\right)$ on $H^{*}\left(\Omega S^{n}\right)$. Applying naturality of $\Phi_{0}$ to the map $F_{2^{k}-1} \rightarrow \Omega S^{n}$ gives us the same result for $H^{*}\left(F_{2^{k}-1}\right)$. Also, the Steenrod operations annihilate $\gamma_{1}\left(\rho_{i}\right)$, so $\Sigma \gamma_{1}\left(\rho_{i}\right)$ is annilated by the Steenrod operations. In particular, $\Phi_{0}$ is defined on $\gamma_{1}\left(\rho_{i}\right)$. We have a commutative diagram.

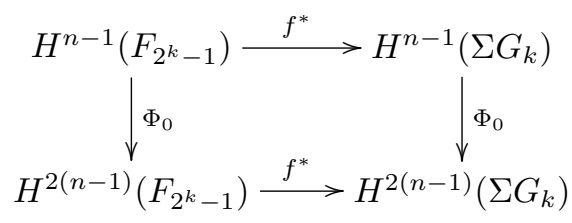

Thus, $f^{*}\left(\Phi_{0}\left(\gamma_{1}\left(\alpha_{n-1}\right)\right)\right)=\Phi_{0}\left(f^{*}\left(\gamma_{1}\left(\alpha_{n-1}\right)\right)\right)$, and so modulo the indeterminacy of $\Phi_{0}$ on $H^{*}\left(\Sigma G_{k}\right)$, we have

$$
\Phi_{0}\left(\Sigma \gamma_{1}\left(\rho_{0}\right)\right)=\Sigma \gamma_{1}\left(\rho_{1}\right) .
$$

As $\gamma_{1}\left(\rho_{1}\right)$ is not in the image of the Steenrod algebra action on $H^{*}\left(G_{k}\right), \Sigma \gamma_{1}\left(\rho_{1}\right)$ is not in the image of the Steenrod algebra action on $H^{*}\left(\Sigma G_{k}\right)$. Recall the suspension homomorphism $s_{l}$ has the property that $s_{2^{i}(n-1)-1}\left(\Sigma \gamma_{1}\left(\rho_{i}\right)\right)=\gamma_{1}\left(\rho_{i}\right)$. Following the discussion of $\sigma \Phi$ in Section 3.1, we have

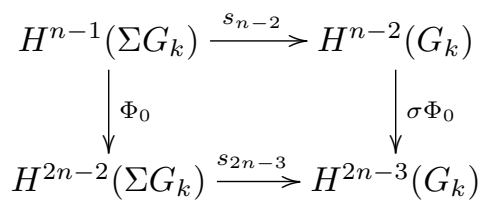


Then,

$$
s_{2 n-3}\left(\sigma \Phi_{0}\left(\Sigma \gamma_{1}\left(\rho_{0}\right)\right)\right)=\Phi_{0}\left(s_{n-2}\left(\Sigma \gamma_{1}\left(\rho_{0}\right)\right) .\right.
$$

So, modulo the indeterminacy of $\sigma \Phi_{0}$ on $H^{*}\left(G_{k}\right)$,

$$
\sigma \Phi_{0}\left(\gamma_{1}\left(\rho_{0}\right)\right)=\gamma_{1}\left(\rho_{1}\right)
$$

Since $\gamma_{1}\left(\rho_{1}\right)$ is not in the image of the Steenrod algebra action on $H^{*}\left(G_{k}\right), \gamma_{1}\left(\rho_{1}\right)$ is not in the indeterminacy module of $\sigma \Phi_{0}$ on $H^{*}\left(G_{k}\right)$.

We have the following naturality diagram for $k=2$, observing that the Steenrod algebra action on $\gamma_{1}\left(\rho_{0}\right) \in H^{*}\left(\Omega^{2} S^{n}\right)$ enables $\sigma \Phi_{0}\left(\gamma_{1}\left(\rho_{0}\right)\right)$ to be defined:

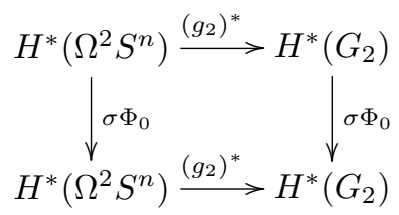

Then, modulo the indeterminacy of $\sigma \Phi_{0}$ on $H^{*}\left(\Omega^{2} S^{n}\right)$,

$$
\begin{aligned}
\left(\left(g_{2}\right)_{*}\right)\left(\sigma \Phi_{0}\left(\gamma_{1}\left(\rho_{0}\right)\right)\right) & =\sigma \Phi_{0}\left(\left(g_{2}\right)_{*}\left(\gamma_{1}\left(\rho_{0}\right)\right)\right) \\
& =\sigma \Phi_{0}\left(\gamma_{1}\left(\rho_{0}\right)\right) \\
& =\gamma_{1}\left(\rho_{1}\right) .
\end{aligned}
$$

Using the naturality argument from Section 2.3 gives the result that $r_{1}$ is not spherical.

7.3.4. Showing $r_{1}$ is not spherical when $n$ is a power of 2

We take the case where $n=2^{r+1}$ where $r \geqslant 3$. Then, in Section 4.3, we have shown that a tertiary cohomology operation $\Psi$ exists such that

$$
\Psi\left(\gamma_{1}\left(\alpha_{n-1}\right)\right)=\gamma_{2}\left(\alpha_{n-1}\right)
$$

with zero indeterminacy on $H^{*}\left(\Omega S^{n}\right)$. By naturality, we have

$$
\Psi\left(\gamma_{1}\left(\alpha_{n-1}\right)\right)=\gamma_{2}\left(\alpha_{n-1}\right)
$$

on $H^{*}\left(F_{2^{k}-1}\right)$ for $k \geqslant 2$ with zero indeterminacy.

We must check that Adams secondary cohomology operations $\Phi_{i j}$ with $i, j \leqslant r$ evaluate to zero on $\Sigma \gamma_{1}\left(\rho_{0}\right) \in H^{*}\left(\Sigma G_{2}\right)$. We have the commutative diagram,

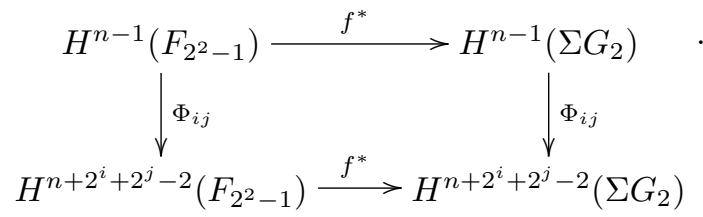

Then, modulo the indeterminacy of $\Phi_{i j}$ on $H^{*}\left(\Sigma G_{2}\right)$, we have

$$
\Phi_{i j}\left(f^{*}\left(\gamma_{1}\left(\alpha_{n-1}\right)\right)\right)=f^{*}\left(\Phi_{i j}\left(\gamma_{1}\left(\alpha_{n-1}\right)\right)\right)=0 .
$$


Homology, Homotopy and Applications, vol. 9(1), 2007

For each $i, j$, the indeterminacy of $\Phi_{i j}$ takes the form

$$
\Sigma_{m} b_{m} H^{2^{i}+2^{j}-1+n-1-\left|b_{m}\right|}\left(\Sigma G_{2}\right)
$$

where $b_{m}$ are Steenrod operations of degree larger than 0. A lower bound for

$$
2^{i}+2^{j}-1+n-1-\left|b_{m}\right|
$$

is $n-1$, and an upper bound is $2 n-3$. By dimensional arguments, we see only two elements of $H^{*}\left(\Sigma G_{2}\right)$ have dimensions falling within these bounds, namely $\Sigma \gamma_{1}\left(\rho_{0}\right)$ and $\Sigma \gamma_{2}\left(\rho_{0}\right)$ with dimensions $n-1$ and $2 n-3$ respectively. However all Steenrod operations annihilate these elements when $n$ is even. Thus, it must be the case that the module of indeterminacy is zero. So, with zero indeterminacy,

$$
\Phi_{i j}\left(\Sigma \gamma_{1}\left(\rho_{0}\right)\right)=0 .
$$

Then, $\Psi$ is defined on $\Sigma \gamma_{1}\left(\rho_{0}\right)$. We have the commutative diagram

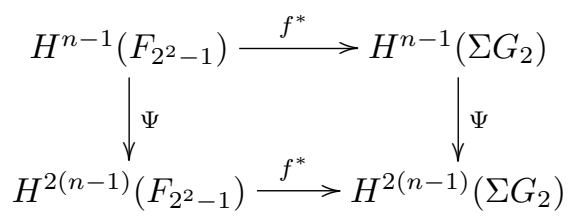

Thus, $\Psi\left(f^{*}\left(\gamma_{1}\left(\alpha_{n-1}\right)\right)\right)=f^{*}\left(\Psi\left(\gamma_{1}\left(\alpha_{n-1}\right)\right)\right)=\Sigma \gamma_{1}\left(\rho_{1}\right)$. That is, modulo indeterminacy,

$$
\Psi\left(\Sigma \gamma_{1}\left(\rho_{0}\right)\right)=\Sigma \gamma_{1}\left(\rho_{1}\right) .
$$

We observe that $\Sigma \gamma_{1}\left(\rho_{1}\right)$, with dimension $2 n-2$, is not in the image of Steenrod operations, so $\Sigma \gamma_{1}\left(\rho_{1}\right)$ is not in the indeterminacy for $\Psi$. Using dimensional criteria, the only other candidates in the indeterminacy are Steenrod operations applied to $\Sigma \gamma_{1}\left(\rho_{0}\right)$ and $\Sigma \gamma_{2}\left(\rho_{0}\right)$. Since Steenrod operations annihilate these elements, the indeterminacy is zero.

Since $\Phi_{i j}$ is stable, $\Phi_{i j}\left(\gamma_{1}\left(\rho_{0}\right)\right)=0$ on $H^{*}\left(G_{2}\right)$. Thus $\Psi$ is defined on $\gamma_{1}\left(\rho_{0}\right)$. Then, we have the commutative diagram

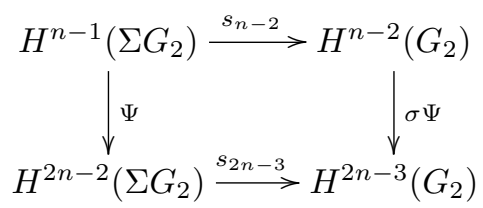

So,

$$
\sigma \Psi\left(\gamma_{1}\left(\rho_{0}\right)\right)=\sigma \Psi\left(s_{n-2}\left(\Sigma \gamma_{1}\left(\rho_{0}\right)\right)\right)=s_{2 n-3}\left(\Psi\left(\Sigma \gamma_{1}\left(\rho_{0}\right)\right)\right)=\gamma_{1}\left(\rho_{1}\right) .
$$

Now, $\left.\gamma_{1}\left(\rho_{1}\right)\right)$ is not in the image of the Steenrod action on $H^{*}\left(\Omega^{2} S^{n}\right)$. Thus, using naturality of $\sigma \Psi$ with respect to $g_{2}$ yields the relation (7.4), considering $\gamma_{1}\left(\rho_{0}\right)$ and $\gamma_{1}\left(\rho_{1}\right)$ as elements of $H^{*}\left(\Omega^{2} S^{n}\right)$. Section 2.3 shows that $r_{1}$ is not spherical. Thus, we have shown that $\Omega^{2} S^{n}$ is minimal atomic for $n \geqslant 6$ unless $n=8,9$. 
Homology, Homotopy and Applications, vol. 9(1), 2007

\subsection{The cases where $\Omega^{2} S^{n}$ is not minimal atomic}

We show that $\Omega^{2} S^{n}$ is not minimal atomic for $n=2,3,4,5,8,9$. Recall that $\left(x_{1}\right)^{2} \in H_{2}\left(\Omega S^{2}\right),\left(x_{3}\right)^{2} \in H_{6}\left(\Omega S^{4}\right)$, and $\left(x_{7}\right)^{2} \in H_{14}\left(\Omega S^{8}\right)$.

\subsection{1. $n=3,5,9$}

We take the case where $n=3,5,9$. Here we will show there exists a spherical class with degree above the Hurewicz dimension for $\Omega^{2} S^{3}, \Omega^{2} S^{5}$, and $\Omega^{2} S^{9}$. Consider the canonical map, $S^{n-1} \stackrel{\eta}{\rightarrow} \Omega S^{n}$. Here, $i_{n-1} \in H_{n-1}\left(S^{n-1}\right)$ maps to $a_{n-1}$ where $H_{*}\left(\Omega S^{n}\right)=\mathbb{F}_{2}\left[a_{n-1}\right]$. For each $n$ we have shown there exists $f: S^{2 n-1} \rightarrow \Omega S^{n-1}$ such that $i_{2(n-1)}$ maps to $a_{n-2}^{2}$. We hope to understand the map $\Omega S^{n-1} \stackrel{\Omega \eta}{\rightarrow} \Omega^{2} S^{n}$. Recall that $H_{*}\left(\Omega^{2} S^{n}\right)=\mathbb{F}_{2}\left[r_{0}, r_{1}, r_{2}, \ldots\right]$ where $\left|r_{i}\right|=2^{i}(n-1)-1$.

Let $' E$ be the homology Serre spectral sequence for the fibration $\Omega S^{n-1} \rightarrow * \rightarrow$ $S^{n-1}$, and let $E$ be the homology Serre spectral sequence for the fibration $\Omega^{2} S^{n} \rightarrow$ $* \rightarrow \Omega S^{n}$. The canonical map $\eta$ induces a map $' E \rightarrow E$ of spectral sequences.

Then, $\Omega \eta$ has the property that

$$
(\Omega \eta)_{*}\left(a_{n-2}\right)=r_{0}
$$

and

$$
(\Omega \eta)_{*}\left(a_{n-2}\right)^{2}=\left(r_{0}\right)^{2}
$$

Thus, in the composition

$$
S^{2(n-2)} \stackrel{f}{\rightarrow} \Omega S^{n-1} \stackrel{\Omega \eta}{\rightarrow} \Omega^{2} S^{n}
$$

we have

$$
i_{2(n-2)} \stackrel{f_{*}}{\rightarrow}\left(a_{n-2}\right)^{2} \stackrel{(\Omega \eta)_{*}}{\rightarrow}\left(r_{0}\right)^{2} .
$$

It follows that $\left(r_{0}\right)^{2}$ is spherical and $\Omega^{2} S^{3}, \Omega^{2} S^{5}$ and $\Omega S^{9}$ are not minimal atomic. Observe that in the cases where $\Omega^{2} S^{2^{q}-1}$ is minimal atomic, our proof in Section 7.2.3 focused on showing that the element $\left(r_{0}\right)^{2}$ is not spherical.

7.4.2. $n=2,4,8$

We show that for the cases $n=2,4,8, \Omega^{2} S^{n}$ is not minimal atomic. For each $n$ we have shown there exists $f: S^{2 n-1} \rightarrow \Omega S^{n}$ with $i_{2(n-1)}$ mapping to $\left(a_{n-1}\right)^{2}$. Looping $f$, we obtain

$$
\Omega S^{2(n-1)} \stackrel{\Omega f}{\rightarrow} \Omega^{2} S^{n}
$$

Now, $H_{*}\left(\Omega S^{2(n-1)}\right)=\mathbb{F}_{2}\left[a_{2(n-1)-1}\right]$. Let $' E$ be the homology Serre spectral sequence for the fibration $\Omega S^{2(n-1)} \rightarrow * \rightarrow S^{2(n-1)}$, and let $E$ be the homology Serre spectral sequence for the fibration $\Omega^{2} S^{n} \rightarrow * \rightarrow \Omega S^{n}$. The map $f$ induces a map ' $E \rightarrow E$ of spectral sequences, which allows us to see that $(\Omega f)_{*}\left(a_{2(n-1)-1}\right)=r_{1}$. Let

$$
g: S^{2(n-1)-1} \rightarrow \Omega S^{2(n-1)}
$$

be the non-trivial map such that $i_{2(n-1)-1} \in H_{*}\left(S^{2(n-1)-1}\right)$ maps to $a_{2(n-1)-1}$ under $g_{*}$. Then the composite, 
Homology, Homotopy and Applications, vol. 9(1), 2007

$$
S^{2(n-1)-1} \stackrel{g}{\rightarrow} \Omega S^{2(n-1)-1} \stackrel{\Omega f}{\rightarrow} \Omega^{2} S^{n}
$$

maps the fundamental class to $r_{1}$. Thus, $r_{1}$ is spherical and $\Omega^{2} S^{2}, \Omega^{2} S^{4}$ and $\Omega^{2} S^{8}$ are not minimal atomic. Looking back on our proof in Section 7.3.4, observe that we had to introduce a special argument to show that $r_{1}$ is not spherical in the cases where $n$ is a power of 2 greater than 8 .

\section{Appendix A. Factoring $S q^{2^{r+1}}$}

Let $r \geqslant 3$. For any space $X$, let $\tau \in H^{*}(X)$ be such that $S q^{2^{s}}(\tau)=0$ for $0 \leqslant s \leqslant$ $r$. Recall (3.3), which states that there exist Steenrod operations $a_{i j}$ such that

$$
S q^{2^{r+1}}(\tau)=\sum_{i \leqslant j, i+1 \neq j} a_{i j} \Phi_{i j}(\tau) .
$$

To obtain a specific factorization of $S q^{2^{s}}$ we need to calculate the coefficients $a_{i j}$ that satisfy (3.3). In $[\mathbf{L W}]$, one factorization of $S q^{16}$ is given, but it is worth noting that there are several factorizations of $S q^{16}$ and one factorization might be preferable to another depending on context. A computer program has been implemented in Maple which builds upon [MN]. This new program calculates all $a_{i j}$ which satisfy (3.3). In this appendix, we examine the mathematics of these coefficients discussed in $[\mathbf{A}]$. This provides a constructive approach to finding $a_{i j}$ which forms the basis of the aforementioned computer program. It should be noted that the program does have flaws - it yields $a_{i j}$ for factorizations of $S q^{16}$, but for values of $r$ larger than 3 , the program stalls ostensibly due to memory constraints. Perhaps this can be improved in the future.

Let $C_{0}$ be the free module over the Steenrod algebra with basis element $c$ of degree 1 , and let $\epsilon: C_{0} \longrightarrow \mathbb{Z} / 2 \mathbb{Z}$ be the non-trivial map. Let $C_{1}$ be the free module over the Steenrod algebra with basis elements $c_{i}$ of degree $2^{i}$. Define $d_{1}: C_{1} \rightarrow C_{0}$ by setting

$$
d_{1}\left(c_{i}\right)=S q^{2^{i}}(c) .
$$

We may construct a minimal resolution of $\mathbb{Z} / 2 \mathbb{Z}$ over the Steenrod algebra with these starting terms,

$$
\cdots \longrightarrow C_{s} \stackrel{d_{s}}{\longrightarrow} C_{s-1} \stackrel{d_{s-1}}{\longrightarrow} C_{s-2} \longrightarrow \cdots \longrightarrow C_{1} \stackrel{d_{1}}{\longrightarrow} C_{0} \stackrel{\epsilon}{\longrightarrow} \mathbb{Z} / 2 \mathbb{Z} \longrightarrow 0 .
$$

Let $I(A)=\sum_{q>0} A_{q}$ where $A$ is the Steenrod algebra and $A_{q}$ consists of those elements of $A$ with degree $q$. Then,

$$
\operatorname{Tor}_{s, t}^{A}(\mathbb{Z} / 2 \mathbb{Z}, \mathbb{Z} / 2 \mathbb{Z}) \cong\left(\mathbb{Z} / 2 \mathbb{Z} \otimes_{A} C\right)_{s, t}
$$

and

$$
\operatorname{Ext}_{A}^{s, t}(\mathbb{Z} / 2 \mathbb{Z}, \mathbb{Z} / 2 \mathbb{Z}) \cong \operatorname{Hom}_{A}^{t}\left(C_{s}, \mathbb{Z} / 2 \mathbb{Z}\right) .
$$

Now for each $C_{s}$, let $J\left(C_{s}\right)=I(A) \cdot C_{s}$ and $Z(s)=\operatorname{Ker}\left(d_{s}\right) \cap J\left(C_{s}\right)$. We observe that for any partial minimal resolution, 
Homology, Homotopy and Applications, vol. 9(1), 2007

$$
C_{s} \stackrel{d_{s}}{\longrightarrow} C_{s-1} \stackrel{d_{s-1}}{\longrightarrow} C_{s-2} \longrightarrow \cdots \longrightarrow C_{1} \stackrel{d_{1}}{\longrightarrow} C_{0} \stackrel{\epsilon}{\longrightarrow} \mathbb{Z} / 2 \mathbb{Z} \longrightarrow 0
$$

we may define a homomorphism

$$
\theta: Z(s) \rightarrow \operatorname{Tor}_{s+1}^{A}(\mathbb{Z} / 2 \mathbb{Z}, \mathbb{Z} / 2 \mathbb{Z}) .
$$

by the following: Extend the partial minimal resolution to another partial minimal resolution by adjoining an appropriate $\left(C_{s+1}, d_{s+1}\right)$. For any $z \in Z(s)$, given $w \in C_{s+1}$ such that $d_{s+1}(w)=z$,

$$
\theta(z)=\left\{1 \otimes_{A} w\right\}
$$

Adams shows that we can choose a cycle $z_{i j} \in C_{1}$ with $i \leqslant j$ and $i+1 \neq j$ such that

$$
h_{i} h_{j}\left(\theta z_{i j}\right)=1
$$

where $h_{i}, h_{j}$ are the basis elements of $\operatorname{Ext}_{A}^{1, t}(\mathbb{Z} / 2 \mathbb{Z}, \mathbb{Z} / 2 \mathbb{Z})$ of degree $2^{i}$ and $2^{j}$ respectively. Now, define $C_{2}$ to be the free module over the Steenrod algebra on generators $c_{i, j}$ such that $d_{2}\left(c_{i j}\right)=z_{i j}$. Adams shows that

$$
C_{2} \stackrel{d_{2}}{\longrightarrow} C_{1} \stackrel{d_{1}}{\longrightarrow} C_{0} \stackrel{\epsilon}{\longrightarrow} \mathbb{Z} / 2 \mathbb{Z} \longrightarrow 0
$$

is a partial minimal resolution over $\mathbb{Z} / 2 \mathbb{Z}$ over $A$. Adams continues to show that there must exist $z \in C_{2}$ such that

$$
h_{0} h_{r}^{2}(\theta z)=1
$$

We write $z$ in the $C_{2}$ basis, so that

$$
z=\sum a_{i j} c_{i j}
$$

The coefficients here are the desired $a_{i j}$.

To obtain explicit coefficients $a_{i j}$, we require explicit representations of $z_{i j}$ in the $C_{1}$ basis. Adams describes how to obtain such a representation in $[\mathbf{A}]$. We review the procedure, and check that the result satisfies (A.1) using Lemma A.1 below. This lemma will also be helpful in trying to find an element $z$ which satisfies (A.2).

Let $t^{\prime}$ be a positive integer, and suppose we are given a function $\alpha: A \rightarrow \mathbb{Z} / 2 \mathbb{Z}$ of degree $-t^{\prime}$ such that $\alpha(a b)=0$ for $a, b \in I(A)$. Then the composite

$$
C_{1} \stackrel{d_{1}}{\longrightarrow} A \stackrel{\alpha}{\longrightarrow} \mathbb{Z} / 2 \mathbb{Z}
$$

defines an element $h_{\alpha} \in \operatorname{Ext}_{A}^{1, t^{\prime}}(\mathbb{Z} / 2 \mathbb{Z}, \mathbb{Z} / 2 \mathbb{Z})$. We have the following lemma from $[\mathbf{A}]$.

Lemma A.1. Given any $h \in \operatorname{Ext}_{A}^{s, t}(\mathbb{Z} / 2 \mathbb{Z}, \mathbb{Z} / 2 \mathbb{Z})$ and any $x \in Z(s) \cap C_{s, t+t^{\prime}}$ with $x=\sum a_{i} c_{i}^{\prime}$

$$
\left(h_{\alpha} h\right)(\theta x)=\sum\left(\alpha a_{i}\right)\left(h\left\{1 \otimes_{A} c_{i}^{\prime}\right\}\right)
$$

where $\left\{c_{i}^{\prime}\right\}$ is the basis of $C_{s}$. 
We will show that for each $i, j$ with $i \leqslant j$ and $i+1 \neq j$, there exists an equation of the type,

$$
S q^{2^{i}} S q^{2^{j}}+\sum f_{k} S q^{2^{g_{k}}}=0
$$

Then, it must be the case that

$$
d_{1}\left(S q^{2^{i}} c_{j}+\sum f_{k} c_{g_{k}}\right)=0 .
$$

We set $z_{i j} \in C_{1}$ to be $S q^{2^{i}} c_{j}+\sum f_{k} c_{g_{k}}$ and show using Lemma A.1 that (A.1) is satisfied.

To obtain (A.3), recall the following standard result.

Lemma A.2. For $m \geqslant 1$ with $m$ not a power of 2 , there exist finitely many Steenrod algebra elements $b_{k}$ and non-negative integers $c_{k}$ such that

$$
S q^{m}=\sum b_{k} S q^{2^{c k}}
$$

It is clear that such a decomposition exists since $\left\{S q^{2^{k}}\right\}$ comprises a set of generators for $A$, but the proof that follows reviews a method for obtaining the desired decomposition, which can be easily translated into computer code.

Proof. Let $2^{d}$ be the largest power of 2 which occurs in the binary representation of $m$. Then $2^{d}>m-2^{d}$. In Section 4.2, we saw that an application of the Adem relations to $S q^{m-2^{d}} S q^{2^{d}}$ yields

$$
S q^{m}=S q^{m-2^{d}} S q^{2^{d}}+\sum S q^{l_{k}} S q^{m_{k}}
$$

where $m_{k} \leqslant m-2^{d}<2^{d}$. If we apply a similar factorization to those $S q^{m_{k}}$ for which $m_{k}$ is not a power of 2 , and iterate this process, we will obtain the desired result of Lemma A.2.

To obtain $z_{i j}$ for any $i, j$ with $i \leqslant j$ and $i+1 \neq j$, observe that the Adem relations apply to give a factorization

$$
S q^{2^{i}} S q^{2^{j}}=\sum S q^{m_{k}} S q^{n_{k}}
$$

If $i=j$, then the binomial coefficient of $S q^{2^{i}+2^{j}}$ is $\left(\begin{array}{c}2^{i}-1 \\ 2^{i}\end{array}\right)=0$, so in the factorization above all $m_{k}, n_{k} \neq 0$ and $n_{k}<2^{i}, 2^{j}$.

If $i \neq j$, then the binomial coefficient of $S q^{2^{i}+2^{j}}$ is $\left(\begin{array}{c}2^{j}-1 \\ 2^{i}\end{array}\right)=1$ by (4.1). If we apply the Adem relations to factor $S q^{2^{i+1}} S q^{2^{j}-2^{i}}$, then $S q^{2^{i}+2^{j}}$ appears in the factorization because $\left(2^{j}-2^{i}-1\right)=1$. The other summands in this factorization are of the form $S q^{u_{k}} S q^{v_{k}}$ and $u_{k}, v_{k} \neq 0$ with $v_{k}<2^{i+1}<2^{j}$. Thus, if we add these two factorizations together, the $S q^{2^{i}+2^{j}}$ terms cancel each other out. Thus, we may obtain (after relabelling the $m_{k}$ and $n_{k}$ ) (A.4) above such that $m_{k}, n_{k} \neq 0$ and $n_{k}<2^{j}$. Thus, in each case we have the same kind of factorization of $S q^{2^{i}} S q^{2^{j}}$. We apply Lemma A.2 to each $S q^{n_{k}}$ to rewrite $S q^{2^{i}} S q^{2^{j}}$ as $\sum f_{k} S q^{2^{g_{k}}}$ where $f_{k} \in A$ and $g_{k}<j$ is a non-negative integer. Thus, we have verified (A.3). Further, we can replicate this process using a computer program because the processes are no more difficult than applying the Adem relations multiple times. 
To show $z_{i j}$ satisfies (A.1), consider the Milnor basis element $\xi_{1}^{2^{i}}$ of the dual of A. We apply Lemma A.1 with $\alpha$ taken to be $\xi_{1}^{2^{i}}$ observing that in the notation of that lemma, $h_{\xi_{1}^{2 i}}=h_{i}$. Thus,

$$
h_{i} h_{j}\left(\theta z_{i j}\right)=\xi_{1}^{2^{i}}\left(S q^{2^{i}}\right) h_{j}\left(\left\{1 \otimes c_{j}\right\}\right)+\sum \xi_{1}^{2^{i}}\left(f_{k}\right) h_{j}\left(\left\{1 \otimes c_{g_{k}}\right\}\right)
$$

Now, for dimensional reasons $h_{j}\left(\left\{1 \otimes c_{g_{k}}\right\}\right)=0$. Also for dimensional reasons, $\{1 \otimes$ $\left.c_{j}\right\}$ is the single non-trivial element of $\operatorname{Tor}_{1,2^{j}}^{A}(\mathbb{Z} / 2 \mathbb{Z}, \mathbb{Z} / 2 \mathbb{Z})$, and since $h_{j}$ is the single non-trivial element of $\operatorname{Ext}_{A}^{1,2^{j}}(\mathbb{Z} / 2 \mathbb{Z}, \mathbb{Z} / 2 \mathbb{Z})$, it must be the case that $h_{j}\left(\left\{1 \otimes c_{j}\right\}\right)=$ 1. Thus, $h_{i} h_{j}\left(\theta z_{i j}\right)=1$ and (A.1) is satisfied.

Having shown that a computer program would be able to find explicit examples of $z_{i j}$, we turn to the more complicated issue of how to find $z \in Z(2)$ which satisfies (A.2).

We observe that any $z \in Z(2)$ satisfies (A.2) if and only if $\xi_{1}\left(a_{r r}\right)=1$. Again, in the notation of Lemma A.1, we see that $h_{\xi_{1}}$ is the basis element $h_{0}$. Then,

$$
\left(h_{0} h_{r}^{2}\right)(\theta z)=\sum_{i}\left(\xi_{1} a_{i j}\right)\left(h_{r} h_{r}\left\{1 \otimes_{A} c_{i j}\right\}\right)
$$

For dimensional reasons,

$$
h_{r} h_{r}\left\{1 \otimes_{A} c_{r r}\right\}=1
$$

while for $(i, j) \neq(r, r)$,

$$
h_{r} h_{r}\left\{1 \otimes_{A} c_{i j}\right\}=0 .
$$

Thus, $h_{0} h_{r}^{2}(\theta z)=1$ if and only if $\xi_{1}\left(a_{r, r}\right)=1$. That is,

$$
a_{r r}=S q^{1} \text {. }
$$

Hence, any $z=\sum a_{i j} c_{i j}$ with $z \in Z(2)$ such that $a_{r r}=S q^{1}$, has the property that its coefficents $a_{i j}$ give the desired factorization of $S q^{2^{r+1}}$.

Compared with the information we have regarding $z_{i j}$, the data we have related to $z$ is quite meager. Adams does add to this small repository by observing that our desired $z$ must also satisfy

$$
\xi_{1}^{2^{r}}\left(a_{0 r}\right)=1
$$

Following Lemma A.1, we see that $h_{\xi_{1}^{2 r}}$ is the basis element $h_{r}$. Since the group $\operatorname{Ext}_{A}(\mathbb{Z} / 2 \mathbb{Z}, \mathbb{Z} / 2 \mathbb{Z})$ is commutative $[\mathbf{M T}$, p. 193] we have

$$
1=\left(h_{0} h_{r}^{2}\right)(\theta z)=\left(h_{r}\left(h_{0} h_{r}\right)\right)(\theta z)=\sum_{i}\left(\xi_{1}^{2^{r}} a_{i j}\right)\left(h_{0} h_{r}\left\{1 \otimes_{A} c_{i j}\right\}\right) .
$$

As above, $h_{0} h_{r}\left\{1 \otimes_{A} c_{i j}\right\}$ is non-zero (and equal to 1 ) only when $i=0$ and $j=r$. Then we have that $\xi_{1}^{2^{r}}\left(a_{0 r}\right)=1$ and so,

$$
a_{0 r}=S q^{2^{r}}+a_{2^{r}}
$$

where $a_{2^{r}} \in A_{2^{r}}$ and does not have $S q^{2^{r}}$ as a summand.

Now, the computer program identifies valid $z$ 's by trial and error. It picks different choices of coefficients $a_{i j}$ and tests whether or not the resulting $z$ satisfies the conditions that $z \in Z(2)$ and $h_{0} h_{r}^{2}(\theta z)=1$. The mathematics that we have illustrated 
makes this job a bit easier. Since the degree of $z$ is $2^{r+1}+1$, and $\left|c_{i j}\right|=2^{i}+2^{j}$, it must be that $\left|a_{i j}\right|=2^{r+1}+1-2^{i}-2^{j}$; we restrict the guesses for $a_{i j}$ to be Steenrod operations of the appropriate degree. Further, (A.5) and (A.6) put further constraints on the coefficients $a_{r r}$ and $a_{0 r}$ which allow us to proceed by an educated version of trial and error.

We may write $z_{i j}$ in the $C_{1}$ basis as before with $z_{i j}=S q^{2^{i}} c_{j}+\sum f_{k} c_{g_{k}}$ where $g_{k}<j \leqslant r$. In particular, each $z_{i j}$ is represented in the $C_{1}$ basis using only the basis elements $c_{0}, c_{1}, \ldots, c_{r}$ (and not necessarily all of these basis elements.) Each of the $r+1$ basis elements gives rise to an equation which must be zero: The fact that $\sum a_{i j} z_{i j}=d(z)=0$ is equivalent to stating that the coefficient of each basis element in $\sum a_{i j} z_{i j}$ must sum to zero. This observation plays a role in the computer code. Instead of looking for a whole system of coefficients $a_{i j}$ which simultaneously satisfies our conditions for $z$, the program looks for coefficients one basis element at a time. First, it searches for which $a_{i j}$ actually even appear as coefficients of $c_{r}$ in $\sum a_{i j} z_{i j}$. Then, it uses the previously mentioned trial and error to look for a combination of those $a_{i j}$ which force the sum of coefficients of $c_{r}$ to be zero. Once the program has found coefficients that work it fixes those values of $a_{i j}$ and examines which remaining $a_{i j}$ appear as coefficients of $c_{r-1}$. Using trial and error, the program picks choices for these new coefficients. If the program finds a set of $a_{i j}$ that works for both $c_{r}$ and $c_{r-1}$ it proceeds in the same fashion to look for coefficients of $c_{r-2}$. If it is not able to find coefficients for $c_{r-1}$ which incorporate the previous coefficient settings of $c_{r}$, the program returns to examining $c_{r}$. It looks for a new set of $a_{i j}$ which ensure that the sum of coefficients of $c_{r}$ is zero and continues again. The process eventually outputs a full set of $a_{i j}$ when it finds a complete set of coefficients which has been tested against each basis element $c_{r}$. Then, it repeats the process with untested settings of $a_{i j}$ in order to produce all combinations of $a_{i j}$ which satisfy (3.3).

\section{References}

[A] J.F. Adams. On the non-existence of elements of Hopf invariant one. Ann. of Math. 72 (1960), 69-153.

[AK] J.F. Adams and N.J. Kuhn. Atomic spaces and spectra. Proc. Edinburgh Math. Soc. 32 (1989), 473-481.

[BM] A.J. Baker and J.P. May. Minimal atomic complexes. Topology 43 (2004), 645-665.

[BP] E.H. Brown and F.P. Peterson. Whitehead products and cohomology operations. Quart J. Math. Oxford Ser.(2) 15 (1964), 116-120.

[C] F.R. Cohen. A course in some aspects of classical homotopy theory. Algebraic Topology. Lecture Notes in Mathematics 1286. Springer-Verlag (1987).

[CMN] F.R. Cohen, J.C. Moore, and J.A. Neisendorfer. Exponents in homotopy theory. Algebraic topology and algebraic K-theory. Princeton, N.J., (1983), 3-34. 
[HKM] P. Hu, I. Kriz, and J. P. May. Cores of spaces, spectra and $E_{\infty}$ ring spectra. Homology, Homotopy and Applications 3 (2001), 341-354.

[LW] J.P. Lin and F. Williams. On 6-connected finite $H$-spaces with two torsion. Topology 28 (1989), 7-34.

[KA] T. Kudo and S. Araki. Topology of $H_{n}$-spaces and $H$-squaring operations. Mem. Fac. Sci. Kyusyu Univer. Ser. A. 10 (1956).

[M] J.P. May. A Concise Course in Algebraic Topology. The University of Chicago Press (1999).

[Mc] J. McCleary. A User's Guide to Spectral Sequences. Cambridge University Press (2001).

[MN] K. Monks. Steenrod. Maple software package. Available at http:// math.scranton. edu/monks/software.html.

[MT] R. Mosher and M. Tangora. Cohomology Operations and Applications in Homotopy Theory. Harper \& Row (1968).

[S] P. Selick. Introduction to Homotopy Theory. Field Institute monographs. American Mathematical Society (1997).

[W] G.W. Whitehead. Elements of Homotopy Theory. Springer-Verlag (1978).

[X] K. Xu. Decomposition of $p$-complete $H$-spaces and related problems. Math. Proc. Cambridge Philos. Soc. 138 no. 2 (2005), 263-266.

[Z] A. Zabrodsky. Secondary operations in the cohomology of $H$-spaces. Illinois J. Math. 15 (1971), 648-655.

Rochelle Pereira rmpereira@stkate.edu

Department of Mathematical Sciences

The College of St. Catherine

St. Paul, MN 55105

USA

This article is available at http://intlpress.com/HHA/v9/n1/a1 